\title{
Comprehensive small-signal modeling and Prony analysis-based validation of synchronous interconnected microgrids
}

Naderi, Mobin; Khayat, Yousef; Shafiee, Qobad; Dragicevic, Tomislav; Bevrani, Hassan; Blaabjerg, Frede

Published in:

Energy Reports

Link to article, DOI:

10.1016/j.egyr.2021.10.045

Publication date:

2021

Document Version

Publisher's PDF, also known as Version of record

Link back to DTU Orbit

Citation (APA):

Naderi, M., Khayat, Y., Shafiee, Q., Dragicevic, T., Bevrani, H., \& Blaabjerg, F. (2021). Comprehensive smallsignal modeling and Prony analysis-based validation of synchronous interconnected microgrids. Energy Reports, 7, 6677-6689. https://doi.org/10.1016/j.egyr.2021.10.045

\section{General rights}

Copyright and moral rights for the publications made accessible in the public portal are retained by the authors and/or other copyright owners and it is a condition of accessing publications that users recognise and abide by the legal requirements associated with these rights.

- Users may download and print one copy of any publication from the public portal for the purpose of private study or research.

- You may not further distribute the material or use it for any profit-making activity or commercial gain

- You may freely distribute the URL identifying the publication in the public portal 
Research paper

\title{
Comprehensive small-signal modeling and Prony analysis-based validation of synchronous interconnected microgrids
}

\author{
Mobin Naderi ${ }^{a}$, Yousef Khayat ${ }^{a}$, Qobad Shafiee ${ }^{a}$, Tomislav Dragicevic ${ }^{b}$, Hassan Bevrani ${ }^{a}$, \\ Frede Blaabjerg c,* \\ a Smart/Micro Grids Research Center (SMGRC), University of Kurdistan, Sanandaj, 66177-15175, Kurdistan, Iran \\ ${ }^{\mathrm{b}}$ Department of Electrical Engineering, Technical University of Denmark, Copenhagen, DK 2800 Kgs. Lyngby, Denmark \\ ${ }^{\mathrm{c}}$ Department of Energy Technology, Aalborg University, Aalborg, DK, 9220, Denmark
}

\section{A R T I C L E I N F O}

\section{Article history:}

Received 9 July 2021

Received in revised form 21 September 2021

Accepted 2 October 2021

Available online 18 October 2021

\section{Keywords:}

Interconnected microgrids

Small-signal modeling

Prony analysis

Eigenvalue analysis

Model validation

\begin{abstract}
A B S T R A C T
The small-signal stability of large-scale interconnected microgrids needs to be analyzed to find the most dominant dynamic behaviors. In this paper, a comprehensive and easy-expandable module-based modeling method is proposed, which is expandable to many interconnected AC microgrids through circuit breakers, i.e. synchronous microgrids. Another certain requirement is validating such a large model, which is satisfied using a well-known Prony analysis method. The large-scale interconnected AC microgrids are implemented in a real-time digital simulator to provide input waveforms for the Prony analysis. On the other hand, the dynamic modes of the proposed model are calculated by eigenvalue analysis, and their contributions in each state variable are identified using the participation matrix. In the proposed validation method, the participating modes in each state variable are compared with the natural frequencies of its estimated waveform by Prony analysis. It is concluded that there is a good matching between the participating modes in the state variables and the contributing frequencies in their waveforms that verifies the proposed modeling method.
\end{abstract}

(C) 2021 The Authors. Published by Elsevier Ltd. This is an open access article under the CC BY license (http://creativecommons.org/licenses/by/4.0/).

\section{Introduction}

An individual microgrid (MG) is a self-controllable entity in the modern power grids that comprises a group of distributed energy resources (DERs), storage systems, loads, protection and control devices, which can be operated in both grid-connected and islanded modes. Although DERs can be connected to the grid individually, their integration in MGs improve stability, reliability, economic optimality and allow their higher penetration than individual DERs (Bevrani et al., 2017). Furthermore, these properties can be enhanced by interconnecting individual MGs. This has led to more research interest in interconnected microgrids (IMGs).

The IMGs operating mode is a new operation mode of individual MGs in order to improve their flexibility, reliability, resiliency, DER penetration and also prevent/postpone load shedding by exchanging the power efficiently (Lasseter, Jun. 2011; Shahnia and Arefi, 2017; Shahidehpour et al., 2017). For this purpose, the challenges of IMGs can be studied in different fields. Smallsignal modeling and stability analysis of AC IMGs through circuit breakers (Shahnia and Arefi, 2017; Zhao et al., 2017; Nikolakakos et al., 2016; Shahnia, 2016; Rehimi et al., 2020; Yao et al., 2021),

\footnotetext{
* Corresponding author.

E-mail address: fbl@et.aau.dk (F. Blaabjerg).
}

modeling and stability analysis of AC IMGs through back-to-back converters (Naderi et al., 2019b, 2020b; Sinha et al., 2021; Majumder and Bag, 2014), power oscillation damping and frequency stability enhancement (Nikolakakos et al., 2016; Hossain et al., 2016; Hirase et al., 2020; Ali et al., 2021), and stability improvement by the interlinking devices and DERs control (Zhang et al., 2016; Hao et al., 2018), are the main aspects of the IMGs stability recently studied in the literature. Power exchange control of IMGs is another important issue, which is based on the stability analysis and studies (Wu et al., 2019; Hossain et al., 2016; Golsorkhi et al., 2018; Zamora and Srivastava, 2018). Transient stability of landsea fishery IMGs supplied by seashore energies is also recently studied (Ju et al., 2021).

Stability analysis and improvement of IMGs operation are important due to possible interactions between the individual controllers of IMGs themselves and also the power exchange controllers. In addition, owing to the dominant low-frequency modes of the IMGs, power and frequency oscillations are taken into account (Hossain et al., 2016). Note that the stability analysis, which is usually the small-signal type, is based on a linear model of IMGs. Most of the previous models of IMGs are based on the state space representation (Hao et al., 2018; Zhao et al., 2017; Nikolakakos et al., 2016; Hossain et al., 2016; Shahnia and Arefi, 2017; Shahnia, 2016) where the basis for an autonomous MG is 
firstly presented (Pogaku et al., 2007). There exist new stability analysis methods, which are used for MGs and other power systems such as data-driven stability analysis method (Ferdowsi et al., 2022). Such a method is very preferable for large-scale IMG. Since the order of IMG dynamic model is usually large, its validation is difficult and at the same time necessary. Nevertheless, all the existing models for IMGs are suffering from the lack of comprehensive validation.

Validation methods for linear (small-signal) models can be categorized in three main groups including time-domain, frequency domain and signal analysis-based approaches. The timedomain validation methods compare time-domain waveforms of the model with the same waveforms of the experimental system (Pogaku et al., 2007), the real-time simulated system (Naderi et al., 2019b), a nonlinear model (Shuai et al., 2018) or a detailed model (Nikolakakos et al., 2017). Though the frequency-domain methods are usually used in validating linear systems and comparing large-order models with their reduced-order models, their applications in nonlinear systems are reported (Dragicevic et al., 2019). In these methods, Bode and Nyquist diagrams are used as the comparison tools (de la O Serna et al., 2016; Bevrani, 2014). The signal analysis-based methods, which are taken into account in the system identification are based on the measurement. In fact, the measured data is analyzed to find the inherent features of the signal, e.g. the dynamic modes of the power system frequency. Fourier transform (Bevrani et al., 2014), digital TaylorFourier transform (de la O Serna et al., 2016), eigensystem realization algorithm (TP462, 2012) and Prony analysis (TP462, 2012; Sauer et al., 2017; Zhao and Loparo, 2017; Golpîra et al., 2015; Føyen et al., 2018), are the major signal estimation techniques, which can be used to compare eigenvalues or state variables of the model with the corresponding measured waveforms.

Prony analysis is a viable technique to estimate a signal with a linear sum of damped complex exponentials. In comparison with the similar methods, e.g. Fourier analysis, the Prony method has the benefit of being able to estimate free from a fundamental frequency and their harmonics. The Prony analysis is used as a system identification method in many areas such as power systems (de la O Serna et al., 2016; Føyen et al., 2018; Golpîra et al., 2015). In Føyen et al. (2018), the Prony method is applied to identify the power system oscillations in smart grids. In Bracale et al. (2007), an adaptive Prony method is presented to estimate the harmonics and inter-harmonics of measured current and voltage. In another application, authors employ Prony analysis to fit a reduced-order dynamic equivalence model to a high-order power system in frequency domain (Golpîra et al., 2015). In the literature, a Prony analysis-based validation method is reported for a small-signal FACTS model (Jiang et al., 2006). A 12-bus power system with a unified power flow controller is modeled and the model is validated by comparing eigenvalue and Prony analyses outputs. Note that the Prony analysis can also assess the dynamics of either very large MGs or IMGs, which are constructed from components with unknown dynamic characteristics. Therefore, Prony analysis can serve as a measurement-based alternative dynamics estimation technique when detailed models are not available.

In this paper, a comprehensive and detailed small-signal model of synchronous interconnected MGs via AC lines and circuit breakers is first developed and then validated. The major differences and developments with respect to the existing works are listed as follows.

- A module-based facilitating small-signal modeling method is proposed for large-scale synchronous IMGs, which is expandable for any number of AC MGs interconnected by circuit breakers in different connection structures.
- In the proposed modeling method, all modules are modeled separately, then interconnections among them are considered using the applicable functions of Robust Control Toolbox in MATLAB. In fact, module modeling individually is a simple analytical calculation due to small-scale of modules. In contrast, the interconnecting process, which leads to large-scale IMG systems is a numerical calculation. Whereas, in the exciting works (Zhao et al., 2017; Wu et al., 2019; Nikolakakos et al., 2016), a complete analytical modeling method using several equation substitutions has been presented, where expand-ability and generality for a big number of MGs are hard to be achieved and are not guaranteed.

- A Prony analysis-based validation method is employed for the proposed IMG modeling, which is a powerful signal analyzing method that has been used in conventional and restructured power systems equivalency, identification and validation (TP462, 2012; Sauer et al., 2017; Serna, 2013; Golpîra et al., 2015; Føyen et al., 2018). In the proposed validation method, the dynamic modes, which contribute in each state variable and their participation factors are calculated by the eigenvalue analysis and the participation matrix. On the other hand, the Prony method is employed to access the natural frequencies and their contribution in the waveform of the same state variable, which is obtained from the real-time simulated nonlinear system. The model validation is realized by comparing the dynamic modes/natural frequencies and their contribution in the state variable, which are obtained using Prony and eigenvalue analyses.

- The large-scale AC IMGs are simulated as real time in an OPAL-RT digital simulator. Hence, the practicality of the studied IMGs in the real time is proven, as well as the small-signal model and the real-time simulated IMGs with nonlinear behaviors are compared.

The reminder of this paper is organized as follows. Section 2 gives the small-signal modeling of synchronous IMGs. In Section 3, the Prony analysis-based validation method is proposed for the obtained model. Section 4 addresses the real-time simulation results. Finally, conclusion is given in Section 4 .

\section{Small-signal modeling of interconnected microgrids}

Fig. 1 displays a general structure of synchronous IMGs, including autonomous AC MGs, interlinking lines, as well as circuit breakers as reconfiguration devices. The MGs may have different structures with any number of DERs, loads and lines. Here, the primary and secondary control levels of the hierarchical MG control (Bevrani et al., 2017) are considered, which are shown in Fig. 1. Individual MG control is accomplished by the primary control level and the coordination control among IMGs is attained by the secondary control level (Liu et al., 2017; Ren et al., 2018; Lai et al., 2019; Weng et al., 2020; Lu et al., 2020; Wu et al., 2019; He et al., 2019). Although, the grid-connected operating mode is not of interest in this paper, it is worth mentioning that its required control should be accomplished in the global/tertiary control layer. Thus, the red connections are opened. Connecting circuit breakers are assumed to be fast enough in switching process that their dynamics are neglected in the dynamic modeling. Hence, the main modules to be modeled are AC MGs and interlinking lines. 


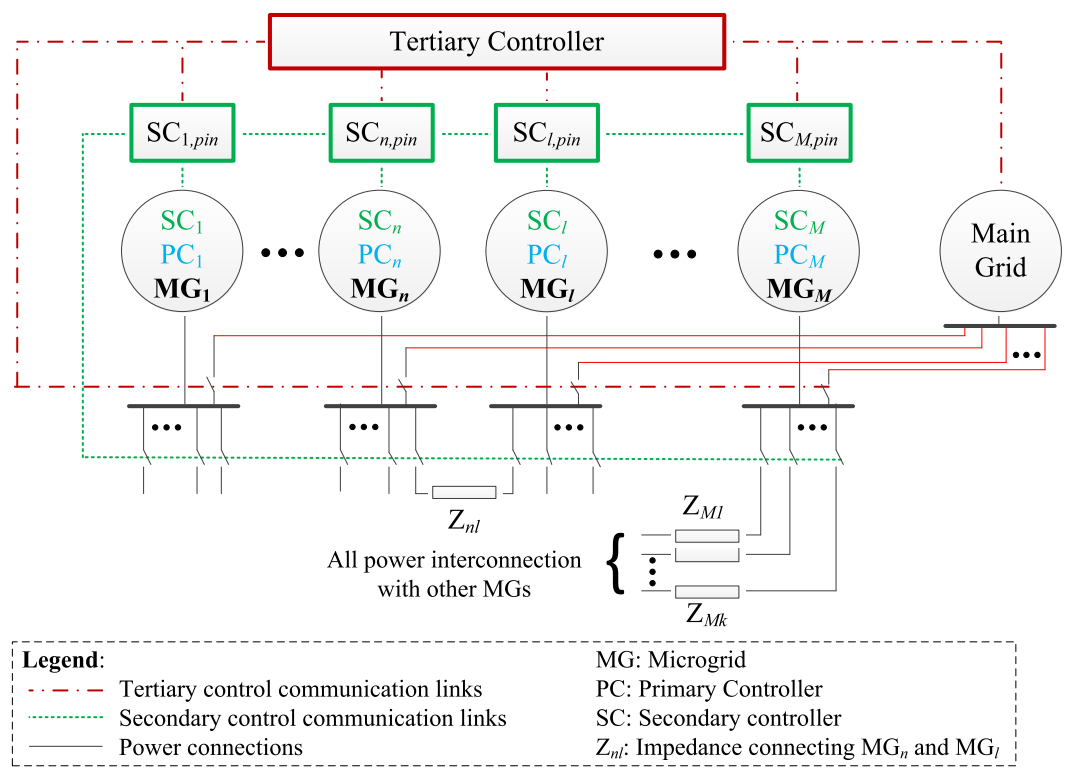

Fig. 1. Synchronous $A C$ microgrids interconnected through $A C$ lines and circuit breakers.

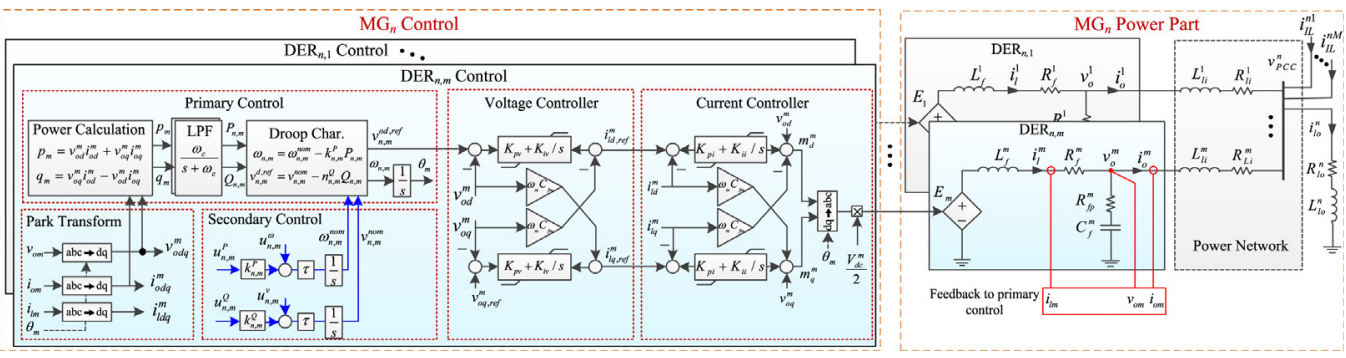

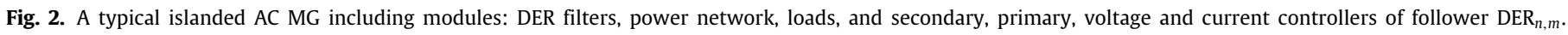

\subsection{Interconnecting process of modules}

In order to find a complete model, the individually modeled modules (sub-models) should be interconnected, which will be a time-consuming and analytically hard process for large-scale systems as shown in Section 2.4. Nevertheless, in the proposed small-signal modeling method, interconnecting modules is accomplished numerically using the useful Robust Control Toolbox functions in MATLAB.

State space representation of modules can be transformed to system matrix form using pck function. The corresponding system matrix forms are considered as sub-models to form the overall system using systemnames function. Function of input_to is used to specify inputs to the sub-models. The inputvar and outputvar functions should be implemented for specifying the desired inputs and outputs of the overall model. Finally, the IMG overall model is obtained by sysic function. Therefore, modeling of any number of synchronous IMGs with different structures can be realized only by interconnecting sub-models of the modules, i.e. MGs and interlinking lines. Furthermore, the proposed module-based method is also used for modeling each autonomous MG, which itself can be a large-scale sub-system.

\subsection{Microgrid modeling}

As shown in Fig. 2, the MG structure consists of the basic elements of AC MGs, including DERs, RLC filters and AC lines. All DERs are assumed as ideal averaging modeled VSCs controlled by the droop-based primary control. The autonomous MGs may be large-scale sub-systems of IMGs, e.g. when they have many number of DERs. Therefore, the module-based modeling method is proposed for finding their individual models. Hence, all MG modules shown in Fig. 2 including secondary, primary, voltage and current controllers, DER power part, power network and MG load are modeled individually. Then, the interconnections among all the MG modules are specified using the Robust Control Toolbox functions. Finding a state space model of each MG is also done by modeling the main modules of the MG and then apply the interconnections like the process presented in 2.1.

\subsubsection{Secondary control level}

In the secondary control level, DERs should communicate some own features to regulate MG voltage and frequency and improve power sharing. One of the most common secondary control methods is the distributed control, which communicate the lowest required data leading to decreasing communication failures (Khayat et al., 2019).

Pinning consensus-based distributed secondary control: In synchronous IMGs, generally, the pinning consensus protocol is used, which is a two-layer communication infrastructure including intra-MG and inter-MG layers (Liu et al., 2017; Ren et al., 2018; Lai et al., 2019; Weng et al., 2020; Lu et al., 2020; Wu et al., 2019; He et al., 2019). For such a nested IMG system with large number of DERs, the pinning consensus-based secondary control technique is much appropriate due to its need to coordination control for a number of DERs, i.e. leader DERs, which leads to reducing the communication costs and failures, and consequently improving reliability. Fig. 3(a), shows a simple 
(a)
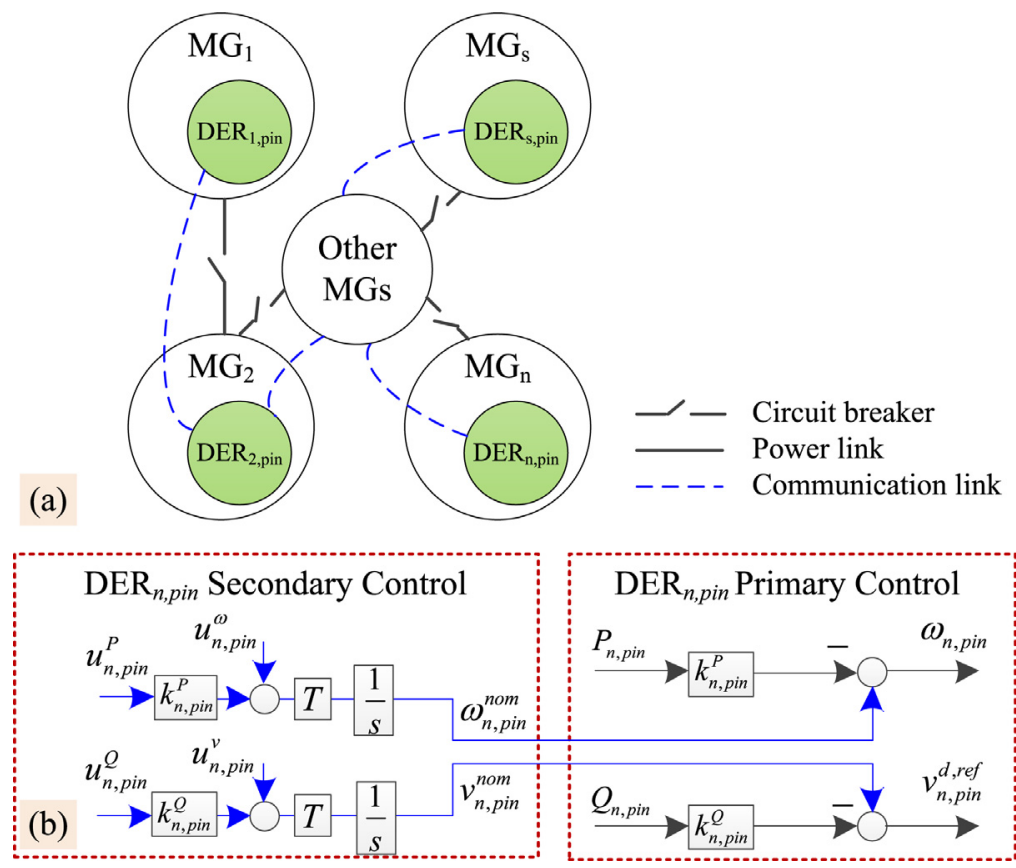

Fig. 3. Pinning consensus secondary control: (a) communication links, (b) control loops.

schematic of the pinning consensus-based distributed secondary control communications including typical inter-MG and intraMG/inter-DER communication links. In each MG, one of the DERs is selected as the leader/pinning/head DER. Therefore, $D_{E R}$ pin as a leader for other follower DERs within the MG to determine the voltage and frequency references, $\omega_{n, p i n}$ and $v_{n, p i n}$, in the intraMG layer as one of its objectives. However, voltage and frequency references for leader DERs themselves are the rated voltage and frequency of MG, i.e. $\omega_{\text {rated }}$ and $v_{\text {rated }}$. Thus, one can consider the leader DER objectives to regulate frequency and voltage as follows:

$\lim _{t \rightarrow \infty}\left|\omega_{\text {rated }}-\omega_{n, p i n}\right|=0, \lim _{t \rightarrow \infty}\left|v_{\text {rated }}-v_{n, p i n}\right|=0$,

$\lim _{t \rightarrow \infty}\left|\omega_{n, p i n}-\omega_{n, m}\right|=0, \lim _{t \rightarrow \infty}\left|v_{n, p i n}-v_{n, m}\right|=0$,

where $n$ and $m$ indicate $n$ 'th MG and $m$ 'th DER, respectively. Note that (1a) states the inter-MG layer requirements, but (1b) belongs to intra-MG layer necessities of frequency/voltage regulation.

$\mathrm{DER}_{p}$ in is also a communicating node in the inter-MG layer to share the information with other MG leaders and thus share the active and reactive powers in IMG level. In fact, in one hand, the leader DERs determine the required powers to be shared among IMGs, and on the other hand, they command the follower DERs to be coordinated to satisfy the IMG power exchanges required for global IMG power sharing. Therefore, the leader DER objectives to share active and reactive powers can be given as follows:

$\lim _{t \rightarrow \infty}\left|k_{n, p i n}^{P} P_{n, p i n}-k_{l, p i n}^{P} P_{l, p i n}\right|=0, \lim _{t \rightarrow \infty}\left|k_{n, p i n}^{Q} Q_{n, p i n}-k_{l, p i n}^{Q} Q_{l, p i n}\right|=0$,

$\lim _{t \rightarrow \infty}\left|k_{n, p i n}^{P} P_{n, p i n}-k_{n, m}^{P} P_{n, m}\right|=0, \lim _{t \rightarrow \infty}\left|k_{n, p i n}^{Q} Q_{n, p i n}-k_{n, m}^{Q} Q_{n, m}\right|=0$,

where $l$ shows $l$ 'th neighbor $\mathrm{MG}$ of $\mathrm{MG}_{n}$. Note that (2a) and (1b) determine inter-MG and intra-MG layers requirements of active/reactive power sharing, respectively. It is worth mentioning that voltage/frequency regulation considerations and active/reactive power sharing requirements of follower DERs in each MG are taken into account in the pinning consensus-based secondary control architecture in a distributed method, which is fully explained in the literature (Khayat et al., 2019).

The distributed secondary controller modeling: As shown in Fig. 2, the secondary controller for both frequency and voltage loops of all DERs is an integral type, where the delay parameters are $\tau$ and $T$ for follower and leader DERs, respectively. The secondary controller outputs are the nominal frequency and voltage, which have also the same form for all DERs. Nevertheless, the secondary control inputs of the leader and follower DERs are different. In fact, the leader DERs receive the rated MG values ( $\omega_{\text {rated }}$ and $\left.v_{\text {rated }}\right)$ and follower DERs receive the MG leader values $\left(\omega_{\text {pin }}\right.$ and $\left.v_{\text {pin }}\right)$. Therefore, the governing relationships of these two DER types are different.

According to Fig. 3(b), for the typical leader/pinning DER of $\mathrm{MG}_{n}$, the dynamic relations are given as follows:

$\dot{\omega}_{n, p i n}^{n o m}=T\left(u_{n, p i n}^{\omega}+k_{n, p i n}^{P} u_{n, p i n}^{P}\right)$,

$\dot{v}_{n, p i n}^{\text {nom }}=T\left(u_{n, p i n}^{v}+k_{n, p i n}^{Q} u_{n, p i n}^{Q}\right)$,

where the input signals are as follows:

$u_{n, p i n}^{\omega}=\sum_{l \in \tilde{N}_{n}} \tilde{a}_{n l}\left(\omega_{l, p i n}-\omega_{n, p i n}\right)+\tilde{a}_{n 0}\left(\omega_{\text {rated }}-\omega_{n, p i n}\right)$,

$u_{n, p i n}^{P}=\left(1 / k_{n, p i n}^{P}\right) \sum_{l \in \tilde{N}_{n}} \tilde{a}_{n l}\left(k_{l, p i n}^{P} P_{l, p i n}-k_{n, p i n}^{P} P_{n, p i n}\right)$,

$u_{n, p i n}^{v}=\sum_{l \in \tilde{N}_{n}} \tilde{a}_{n l}\left(v_{l, p i n}-v_{n, p i n}\right)+\tilde{a}_{n 0}\left(v_{r a t e d}-v_{n, p i n}\right)$,

$u_{n, p i n}^{Q}=\left(1 / k_{n, p i n}^{Q}\right) \sum_{l \in \tilde{N}_{n}} \tilde{a}_{n l}\left(k_{l, p i n}^{Q} Q_{l, p i n}-k_{n, p i n}^{Q} Q_{n, p i n}\right)$.

The inter-MG cyber network can be defined as $\tilde{\chi}(\tilde{\vartheta}, \tilde{\varepsilon}, \tilde{A})$ with leader node set $\tilde{\vartheta}=\left\{\vartheta_{1, \text { pin }}, \ldots, \vartheta_{n \text {,pin }}, \ldots, \vartheta_{M, p i n}\right\}$, set of edges $\tilde{\varepsilon} \subseteq \tilde{\vartheta} \times \tilde{\vartheta}$, i.e. communication links among IMGs, and adjacency matrix $\tilde{A}=\left(\tilde{a}_{n l}\right)_{M \times M}$, where $\tilde{a}_{l l}=0$ and $\tilde{a}_{n l} \geqslant 0 . \tilde{a}_{n l}=1$ if and only if $\left(\vartheta_{n, p i n}, \vartheta_{l, p i n}\right) \in \tilde{\varepsilon}$. The neighbors of $\mathrm{MG}_{n}$ is expressed in the set $\tilde{N}_{n}=\left\{\vartheta_{l, p i n} \in \tilde{\vartheta} \mid\left(\vartheta_{n, p i n}, \vartheta_{l, p i n}\right) \in \tilde{\varepsilon}\right\}$. The access of leader DERs to the rated MG values is specified by the leader-adjacency matrix 
$\tilde{B}=\operatorname{diag}\left\{\tilde{a}_{10}, \ldots, \tilde{a}_{n 0}, \ldots, \tilde{a}_{M 0}\right\}$, where $\tilde{a}_{n 0}=1$ if the rated values are available for the $\mathrm{MG}_{n}$ head, otherwise, $\tilde{a}_{n 0}=0$.

According to Fig. 2, for the typical follower $\mathrm{DER}_{n, m}$, the dynamic relations are as follows:

$\dot{\omega}_{n, m}^{n o m}=\tau\left(u_{n, m}^{\omega}+k_{n, m}^{P} u_{n, m}^{P}\right)$,

$\dot{v}_{n, m}^{n o m}=\tau\left(u_{n, m}^{v}+k_{n, m}^{Q} u_{n, m}^{Q}\right)$,

where the input signals are as follows:

$$
\begin{aligned}
u_{n, m}^{\omega}= & \sum_{j \in N_{n, m}} a_{m j}^{n}\left(\omega_{n, j}-\omega_{n, m}\right)+a_{m 0}^{n}\left(\omega_{n, p i n}-\omega_{n, m}\right), \\
u_{n, m}^{P}= & \left(1 / k_{n, m}^{P}\right) \sum_{j \in N_{n, m}} a_{m j}^{n}\left(k_{n, j}^{P} P_{n, j}-k_{n, m}^{P} P_{n, m}\right) \\
& +a_{m 0}^{n}\left(k_{n, p i n}^{P} P_{n, p i n}-k_{n, m}^{P} P_{n, m}\right), \\
u_{n, m}^{v}= & \sum_{j \in N_{n, m}} a_{m j}^{n}\left(v_{n, j}-v_{n, m}\right)+a_{m 0}^{n}\left(v_{n, p i n}-v_{n, m}\right), \\
u_{n, m}^{Q}= & \left(1 / k_{n, m}^{Q}\right) \sum_{j \in N_{n, m}} a_{m j}^{n}\left(k_{n, j}^{Q} Q_{n, j}-k_{n, m}^{Q} Q_{n, m}\right) \\
& +a_{m 0}^{n}\left(k_{n, p i n}^{Q} Q_{n, p i n}-k_{n, m}^{Q} Q_{n, m}\right),
\end{aligned}
$$

The inter-DER cyber network in each $\mathrm{MG}_{n}$ can be defined as $\chi_{n}\left(\vartheta_{n}, \varepsilon_{n}, A_{n}\right)$, which describes the communication interactions among $S-1$ follower DERs, where $S$ indicates the total number of DERs of each MG. The node set $\vartheta_{n}=$ $\left\{\vartheta_{n, 1}, \ldots, \vartheta_{n, p i n-1}, \vartheta_{n, p i n+1}, \ldots, \vartheta_{n, S_{n}}\right\}$ represents the follower DERs, the set of edges $\varepsilon_{n} \subseteq \vartheta_{n} \times \vartheta_{n}$, expresses the communication links among the DERs, and $A_{n}=\left(a_{m j}^{n}\right)_{\left(S_{n}-1\right) \times\left(S_{n}-1\right)}$ is an adjacency matrix, where $a_{m m}^{n}=0$ and $a_{m j}^{n} \geqslant 0 . a_{m j}^{n}=1$ if and only if $\left(\vartheta_{n, m}, \vartheta_{n, j}\right) \in \varepsilon_{n}$. The neighbor set of $\mathrm{MG}_{n}$ can be expressed by $N_{n, m}=\left\{\vartheta_{n, j} \in \vartheta_{n} \mid\left(\vartheta_{n, m}, \vartheta_{n, j}\right) \in \varepsilon_{n}\right\}$. The access of each DER to the $\mathrm{MG}_{n}$ leader values is specified by the leader-adjacency matrix $B_{n}=\operatorname{diag}\left\{a_{10}^{n}, \ldots, a_{m 0}^{n}, \ldots, a_{s 0}^{n}\right\}$, where $a_{m 0}^{n}=1$ if the leader DER values are available for the $\mathrm{DER}_{n, m}$, otherwise, $a_{m 0}^{n}=1$.

By specifying the two integrator outputs as the state variables, one can find a state space representation for the secondary controller of each DER as follows:

$\dot{\boldsymbol{X}}_{S C}=\boldsymbol{A}_{S C} \boldsymbol{X}_{S C}+\boldsymbol{B}_{S C 1} \boldsymbol{U}_{S C 1}+\boldsymbol{B}_{S C 2} \boldsymbol{U}_{S C 2}+\boldsymbol{B}_{S C 3} \boldsymbol{U}_{S C 3}$,

$\boldsymbol{Y}_{S C}=\boldsymbol{C}_{S C} \boldsymbol{X}_{S C}$,

where $\boldsymbol{Y}_{S C}=\boldsymbol{X}_{S C}$ and equal $\left[\omega_{n, \text { pin }}^{\text {nom }} v_{n, p i n}^{\text {nom }}\right]^{T}$ for leader DERs, or $\left[\omega_{n, m}^{n o m} v_{n, m}^{n o m}\right]^{T}$ for follower DERs. Input vectors of the leader and follower DERs are tabulated in Table 1. $\boldsymbol{A}_{S C}=\mathbf{0}_{2}$ and $\boldsymbol{C}_{S C}=$ $\mathbf{I}_{2}$. Obviously, the size and content of the input matrices, $\boldsymbol{B}_{\mathrm{SC} 1}$, $\boldsymbol{B}_{S C 2}$, and $\boldsymbol{B}_{S C 3}$, are strongly related to the inter-MG and interDER communication network configurations. Therefore, it is not easy and not necessary to show them for the general situation. However, they can typically be shown for the case study twoIMGs in which each MG has two DERs and DER 2 is the leader DER. The input matrices of $\mathrm{MG}_{1}$ DERs are given as follows:

$$
\begin{aligned}
\mathbf{B}_{S C 1}^{D E R 1} & =\left[\begin{array}{ccccc}
-\tau & 0 & 0 & -\tau k_{1,1}^{P} & 0 \\
0 & -\tau & 0 & 0 & -\tau k_{1,1}^{Q}
\end{array}\right], \\
\mathbf{B}_{S C 3}^{D E R 1} & =\left[\begin{array}{ccccc}
\tau & 0 & 0 & \tau k_{1,2}^{P} & 0 \\
0 & \tau & 0 & 0 & \tau k_{1,2}^{Q}
\end{array}\right], \\
\mathbf{B}_{S C 2}^{D E R 2} & =\left[\begin{array}{ccccc}
T & 0 & 0 & T k_{2,2}^{P} & 0 \\
0 & T & 0 & 0 & T k_{2,2}^{Q}
\end{array}\right], \\
\mathbf{B}_{S C 3}^{D E R 2} & =\left[\begin{array}{ccccc}
-2 T & 0 & 0 & -T k_{1,2}^{P} & 0 \\
0 & -2 T & 0 & 0 & -T k_{1,2}^{Q}
\end{array}\right] .
\end{aligned}
$$

other cases are zero matrices with appropriate sizes. Note that the matrices of $\mathrm{MG}_{2}$ DERs are similar to (8) with appropriate indices for matrices and their content.
Table 1

Input vectors of the state space representation of the leader and follower DERs' secondary controls.

\begin{tabular}{llll}
\hline DER type & $\boldsymbol{U}_{S C 1}$ & $\boldsymbol{U}_{S C 2}$ & $\boldsymbol{U}_{S C 3}$ \\
\hline Leader DER ${ }_{n, p i n}$ & - & Column vector of $\left\{Y_{P C}^{l, p i n} \mid\left(\vartheta_{n, p i n}, \vartheta_{l, p i n}\right) \in \tilde{\varepsilon}\right\}$ & $\boldsymbol{Y}_{P C}^{n, p i n}$ \\
\hline Follower DER $\mathrm{DE}_{n, m}$ & $\boldsymbol{Y}_{P C}^{n, m}$ & Column vector of $\left\{Y_{P C}^{n, j} \mid\left(\vartheta_{n, m}, \vartheta_{n, j}\right) \in \varepsilon_{n}\right\}$ & $\boldsymbol{Y}_{P C}^{n, p i n}$ \\
\hline
\end{tabular}

The communication delay is an important issue in the distributed secondary control, which may has considerable impacts on IMG stability. In the literature, this issue is studied for AC and DC IMGs. Delay-dependent small-signal stability analysis of AC IMGs through circuit breakers is investigated by considering communication delays within a Lyapunov function (Hao et al., 2018). The instability and oscillations are studied in DC IMGs on dc bus voltages due to time delays of communication channels used in distributed controllers (Dong et al., 2018; Mudaliyar et al., 2020). Communication delay (Han et al., 2018), and global/local layer link failure and time delay (Sahoo et al., 2019) are also taken into consideration to verify the corresponding controllers' performance and their transient stability. Therefore, solving the challenges of communication delay such as modeling, stability analysis, and robustness against it, is still of interest and should be taken into account in future works.

\subsubsection{Primary control level}

The DER power controller comprises $\omega-P$ and $V_{d}-Q$ droop characteristics, a power calculation unit, and two low-pass filters (LPFs) as shown in Fig. 2. The conventional droop characteristics have no dynamics. Therefore, in order to find the small-signal model, it is enough to consider the dynamics of LPFs and the integrator of the local voltage phase $\left(\theta_{m}\right)$ producer. The difference of the common and $\mathrm{DER}_{m}$ reference frames angles, i.e. $\delta_{m}$, is considered as the state variable instead of the $\theta_{m}$ due to the reason presented in Section 2.2.8. It is given as follows:

$\delta_{m}=\theta_{m}-\theta_{\text {com }}^{n}=\int\left(\omega_{m}-\omega_{c o m}^{n}\right) d t$,

where $\omega_{m}$ is the $\mathrm{DER}_{m}$ angular frequency and $\theta_{\text {com }}^{n}$ is the $\mathrm{DER}_{1}$ voltage phase in each MG, and the common reference frame (CRF) is considered to be based on it. Then, a state space representation can be given as:

$\dot{\boldsymbol{X}}_{P C}^{m}=\boldsymbol{A}_{P C}^{m} \boldsymbol{X}_{P C}^{m}+\boldsymbol{B}_{P D P}^{m} \boldsymbol{U}_{P D P}^{m}+\boldsymbol{B}_{P S C}^{m} \boldsymbol{U}_{P S C}^{m}+\boldsymbol{B}_{P C}^{m} \Delta \omega_{c o m}^{n}$,

$\boldsymbol{Y}_{P C}^{m}=\boldsymbol{C}_{P C}^{m} \boldsymbol{X}_{P C}^{m}$,

where $\boldsymbol{X}_{P C}^{m}=\left[\Delta \delta_{m} \Delta P_{m} \Delta Q_{m}\right]^{T}, \boldsymbol{U}_{P D P}^{m}=\boldsymbol{Y}_{D P}^{m}, \boldsymbol{U}_{P S C}^{m}=\boldsymbol{Y}_{S C}$, $\boldsymbol{Y}_{P C}^{m}=\left[\Delta \omega_{m} \Delta \boldsymbol{v}_{d, r e f}^{m} \Delta \delta_{m} \Delta P_{m} \Delta Q_{m}\right]^{T}$ and the matrices are easy to be found (Naderi et al., 2019b).

In general, $f_{d}^{m}$ and $f_{q}^{m}$ are the $d q$-components of $2 \times 1$ vector $\boldsymbol{f}_{m}$, where $f$ can represent each voltage, current, or control variable. 0 at subscripts indicates a variable value at the equilibrium point. All parameters and variables are specified in Fig. 2.

\subsubsection{Voltage controller}

A voltage controller locally regulates DER output voltage, which is usually done in rotary reference frame. The reference of the direct voltage component is reached from the $V_{d}-Q$ droop characteristic and the reference of the quadrature voltage component is usually considered as zero. PI controllers are usual for both voltage and current controllers as shown in Fig. 2. Therefore, a state space representation is easy to be obtained as follows:

$\dot{\boldsymbol{X}}_{V C}^{m}=\boldsymbol{A}_{V C}^{m} \boldsymbol{X}_{V C}^{m}+\boldsymbol{B}_{V C}^{m} \boldsymbol{U}_{V C}^{m}$, 
$\boldsymbol{Y}_{V C}^{m}=\boldsymbol{C}_{V C}^{m} \boldsymbol{X}_{V C}^{m}+\boldsymbol{D}_{V C}^{m} \boldsymbol{U}_{V C}^{m}$,

where $\boldsymbol{X}_{V C}^{m}$ is a $2 \times 1$ vector of the integrators outputs, $\boldsymbol{U}_{V C}^{m}=$ $\left[\begin{array}{ll}\boldsymbol{Y}_{D P}^{m} & \boldsymbol{Y}_{P C}^{m}\end{array}\right]^{T}$ and $\boldsymbol{Y}_{V C}^{m}=\boldsymbol{i}_{l d q}^{m}$. The matrices can be calculated after converting Laplace-domain relationships attained from Fig. 2 to the first-order differential equations and then implementing the modeling steps.

\subsubsection{Current controller}

RLC filter-based DERs require a fast current limiting process, which is realized by restraining the filter current through the current controller. Using a similar structure to the PI voltage controller as shown in Fig. 2 the state space model can be represented as:

$\dot{\boldsymbol{X}}_{C C}^{m}=\boldsymbol{A}_{C C}^{m} \boldsymbol{X}_{C C}^{m}+\boldsymbol{B}_{C C}^{m} \boldsymbol{U}_{C C}^{m}$,

$\boldsymbol{Y}_{C C}^{m}=\boldsymbol{C}_{C C}^{m} \boldsymbol{X}_{C C}^{m}+\boldsymbol{D}_{C C}^{m} \boldsymbol{U}_{C C}^{m}$,

where $\boldsymbol{X}_{C C}^{m}$ is a $2 \times 1$ vector of the integrators outputs, $\boldsymbol{U}_{C C}^{m}=$ $\left[\begin{array}{ll}\boldsymbol{Y}_{D P}^{m} & \boldsymbol{Y}_{V C}^{m} \boldsymbol{Y}_{P C}^{m}\end{array}\right]^{T}, \boldsymbol{Y}_{C C}^{m}=\boldsymbol{m}_{d q}^{m}$, and the matrices can be found in the same way explained for the voltage controller.

\subsubsection{DER power part}

According to Fig. 2 and using common modeling steps comprising to employ circuit laws, Park transformation, $d q$ decoupling, and Taylor series-based linearization, the dynamics of each DER power part, e.g. $\mathrm{DER}_{m}$, including an ideal voltage source and an RLC filter can be obtained by the first-order differential equations. By representing the equations in the matrix form, the state space model can be given as:

$\dot{\boldsymbol{X}}_{D P}^{m}=\boldsymbol{A}_{D P}^{m} \boldsymbol{X}_{D P}^{m}+\boldsymbol{B}_{D P}^{m} \boldsymbol{U}_{D P}^{m}+\boldsymbol{B}_{P N}^{m} \boldsymbol{U}_{P N}^{m}$,

$\boldsymbol{Y}_{D P}^{m}=\boldsymbol{C}_{D P}^{m} \boldsymbol{X}_{D P}^{m}+\boldsymbol{D}_{D P}^{m} \boldsymbol{U}_{D P}^{m}+\boldsymbol{D}_{P N}^{m} \boldsymbol{U}_{P N}^{m}$,

where $\boldsymbol{X}_{D P}^{m}=\left[\begin{array}{ll}\Delta \boldsymbol{i}_{l d q}^{m} & \Delta \boldsymbol{v}_{o d q}^{m}\end{array}\right]^{T}$, the control input $\boldsymbol{U}_{D P}^{m}=$ $\left[\begin{array}{ll}\Delta \boldsymbol{E}_{d q}^{m} & \Delta \omega_{m}\end{array}\right]^{T}$, the disturbance input $\boldsymbol{U}_{P N}^{m}=\boldsymbol{i}_{o d q}^{m}$, and one can easily calculate the matrices.

\subsubsection{Power network}

Note that any configuration of MG power network is able to be considered. However, a usual structure including DER coupling lines and one point of common coupling (PCC) is assumed here (see Fig. 2). In fact, more PCCs and their connecting lines can be reduced to the studied structure using Kron reduction (Shuai et al., 2018). Hence, a dynamic model of the $m$ 'th line is achieved after the modeling steps as the following state space representation:

$\dot{\boldsymbol{X}}_{P L}^{m}=\boldsymbol{A}_{P L}^{m} \boldsymbol{X}_{P L}^{m}+\boldsymbol{B}_{L D}^{m} \boldsymbol{U}_{L D}^{m}+\boldsymbol{B}_{L P}^{m} \boldsymbol{U}_{L P}^{m}+\boldsymbol{B}_{L \omega}^{m} \boldsymbol{U}_{L \omega}^{m}$,

$\boldsymbol{Y}_{P L}^{m}=\boldsymbol{C}_{P L}^{m} \boldsymbol{X}_{P L}^{m}$,

where, $\boldsymbol{X}_{P L}^{m}=\boldsymbol{Y}_{P L}^{m}=\Delta \boldsymbol{i}_{\text {odq }}^{m}, \boldsymbol{U}_{L P}^{m}=\Delta \boldsymbol{v}_{p c c, d q}^{m}, \boldsymbol{U}_{L D}^{m}=\boldsymbol{Y}_{D P}^{m}, \boldsymbol{U}_{L \omega}^{m}=$ $\Delta \omega_{\text {com. }} . \Delta \omega_{\text {com }}$ is the perturbed form of the CRF angular frequency, which is addressed in Section 2.2.8. The matrices can be calculated by comparing (14) with the corresponding first-order differential equations.

\subsubsection{MG load}

Here, a lumped series RL load is considered for each MG. In fact, it is an equivalent load after applying Kron reduction to simplify MGs with any number of loads (Shuai et al., 2018; Nikolakakos et al., 2017). Hence, the expressing relationships for the equivalent $\mathrm{MG}_{n}$ load dynamics can be given as:

$\dot{\boldsymbol{X}}_{M L}^{n}=\boldsymbol{A}_{M L}^{n} \boldsymbol{X}_{M L}^{n}+\boldsymbol{B}_{M L C}^{n} \boldsymbol{U}_{M L C}^{n}+\boldsymbol{B}_{M L P}^{n} \boldsymbol{U}_{M L P}^{n}$,

$\boldsymbol{Y}_{M L}^{n}=\boldsymbol{C}_{M L}^{n} \boldsymbol{X}_{M L}^{n}$,

where $\boldsymbol{X}_{M L}^{n}=\boldsymbol{Y}_{M L}^{n}=\Delta \boldsymbol{i}_{l o, d q}^{n}, \boldsymbol{U}_{M L C}^{n}=\Delta \omega_{c o m}^{n}, \boldsymbol{U}_{M L P}^{n}=\Delta \boldsymbol{v}_{p c c, d q}^{n}$, and all matrices can be easily calculated.

\subsubsection{Common reference frame}

Frequency references of DERs are independently produced as shown in Fig. 2 in the primary control level. Due to the stable operation of the droop characteristics, all DER frequencies will be equal in a stable steady state. However, their transients may be different due to different dynamics of the DER frequency loops. Hence, a CRF is considered to model DER frequency interactions during transients (Nikolakakos et al., 2016; Pogaku et al., 2007). In this way, the following four steps are essential.

(i) The CRF should be determined based on one of the DER reference frames. In this paper, the reference frame of the $D_{E R}$ of $M_{1}$ is determined as the CRF.

(ii) As discussed in Section 2.2.2, $\Delta \delta$ is specified as a state variable in the power controller of each DER.

(iii) $\omega_{\text {com }}$ is employed in modeling modules anywhere that DERs frequencies are not used in modeling, e.g. power networks.

(iv) The output variables of each DER reference frame to the modules stated in the CRF, must be converted into the CRF and vice versa. For example, the $\boldsymbol{v}_{o, d q}^{m}$ is originally presented in the $\mathrm{DER}_{m}$ individual reference frame, and it is an input to the power network sub-model, which is stated in the CRF. Hence, the $\boldsymbol{v}_{o, d q}^{m}$ should be converted into the CRF. Inversely, $\boldsymbol{i}_{o d q}^{m}$ should be converted from the CRF to the $D_{E R}$ reference frame. Both the individual-to-CRF and CRF-to-individual transformations in a perturbed form after linearization are given as follows:

$\begin{aligned} \Delta \boldsymbol{v}_{D Q} & =\boldsymbol{T}_{s} \cdot \Delta \boldsymbol{v}_{d q}+\boldsymbol{T}_{\delta 1} \cdot \Delta \delta, \\ \Delta \boldsymbol{v}_{d q} & =\boldsymbol{T}_{s}^{-1} \cdot \Delta \boldsymbol{v}_{D Q}+\boldsymbol{T}_{\delta 2} \cdot \Delta \delta,\end{aligned}$

where $\boldsymbol{v}_{D Q}$ and $\boldsymbol{v}_{d q}$ indicate variables in CRF and individual frames, respectively. $\boldsymbol{T}_{s}, \boldsymbol{T}_{\delta 1}$ and $\boldsymbol{T}_{\delta 2}$ are reported in Naderi et al. (2019b).

\subsubsection{Overall MG model}

To find the state space representation of overall MG, the interconnections among all modeled modules presented in previous sub-sections should be modeled. The interconnections are based on the MG power and control configurations and can be calculated using the advantageous Robust Control Toolbox functions.

According to Fig. 2, the control loops and power part of $\mathrm{DER}_{m}$ 's are fully modeled by (7), (10), (11), (12), and (13). Moreover, the MG power network and load models are stated using (14) and (15). The PCC voltage $\boldsymbol{v}_{p c c, d q}^{n}$ is as a disturbance input in (14) and (15), which causes lack of solving the set of existing differential equations. Hence, a virtual resistor $r_{v}$ is assumed at the $\mathrm{PCC}_{n}$, which results in the complementary relationship for the set (7), (10), (11), (12), (13), (14), and (15). After adding the virtual resistor and applying $\mathrm{KCL}$ at the $\mathrm{PCC}_{n}$, the PCC voltage is obtained as:

$\boldsymbol{V}_{p c c, d q}^{n}=\sum_{j=1}^{S} \boldsymbol{R}_{V} i_{o d q}^{j}+\sum_{l=1}^{M} \boldsymbol{R}_{V} \boldsymbol{i}_{I L, d q}^{n l}-\boldsymbol{R}_{V} \boldsymbol{i}_{l o, d q}^{n}$,

where, $\boldsymbol{R}_{V}=r_{V} \boldsymbol{I}_{2}$ and $\boldsymbol{i}_{I L, d q}^{n l}$ is the current of $l$ 'th interlinking line connected to the $\mathrm{PCC}_{n}$.

An expandable MG model can be obtained according to Fig. 4, where shows the interconnections of all MG modules. Each MG with any number of DERs, lines and loads can be represented as the state space model:

$\dot{\boldsymbol{X}}_{M G}^{n}=\boldsymbol{A}_{M G}^{n} \boldsymbol{X}_{M G}^{n}+\boldsymbol{B}_{M G}^{n} \boldsymbol{U}_{M G}^{n}$,

$\boldsymbol{Y}_{M G}^{n}=\boldsymbol{C}_{M G}^{n} \boldsymbol{X}_{M G}^{n}+\boldsymbol{D}_{M G}^{n} \boldsymbol{U}_{M G}^{n}$, 


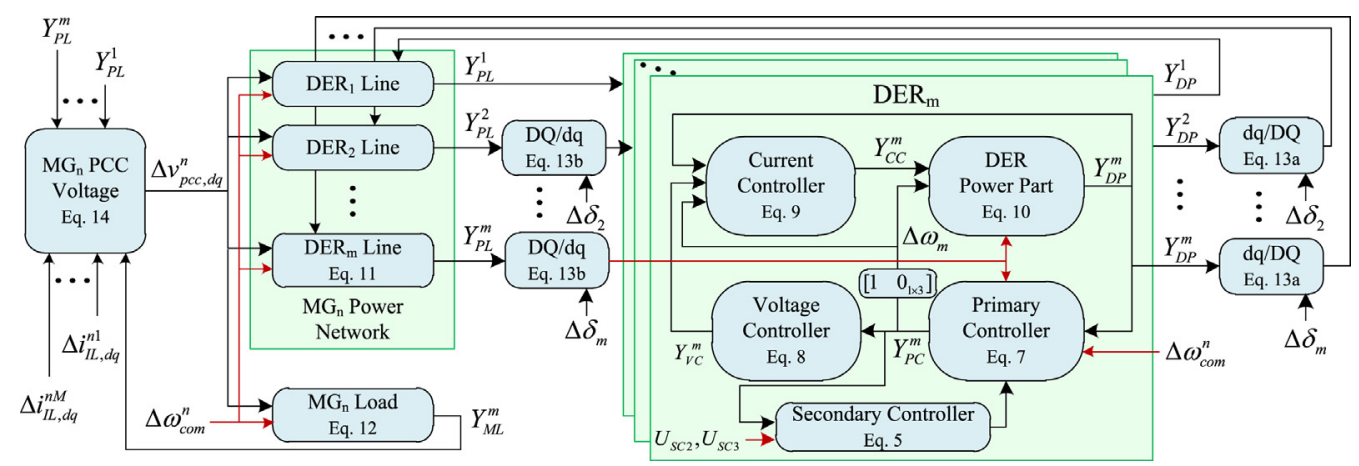

Fig. 4. All power and control interconnections of proposed generalizable modeling for autonomous $A C \mathrm{MG}_{n}$.

where the state vector can be organized as

$$
\begin{aligned}
& \boldsymbol{X}_{D E R}^{m, n} \\
& \boldsymbol{X}_{M G}^{n}=\left[\begin{array}{lllllll}
\boldsymbol{X}_{D E R}^{1, n} & \ldots & \overbrace{\boldsymbol{X}_{S C}^{m}}^{m} \boldsymbol{X}_{P C}^{m} & \boldsymbol{X}_{V C}^{m} & \boldsymbol{X}_{C C}^{m} \boldsymbol{X}_{P D}^{m} & \ldots \boldsymbol{X}_{D E R}^{S, n}
\end{array}\right. \\
& \left.\begin{array}{llllll}
\boldsymbol{X}_{P L}^{1, n} & \ldots & \boldsymbol{X}_{P L}^{m, n} & \ldots & \boldsymbol{X}_{P L}^{S, n} \boldsymbol{X}_{M L}^{n}
\end{array}\right]^{T},
\end{aligned}
$$

and it consists of $15 m+2$ state variables for the MG structure shown in Fig. 2(a), where $m$ is the number of DERs/lines. Interlinking line currents to $\mathrm{MG}_{n}$ are as the disturbance inputs i.e. $\boldsymbol{U}_{M G}^{n}=\left[\begin{array}{lll}\boldsymbol{i}_{I L, d q}^{n 1} & \cdots & \boldsymbol{i}_{I L, d q}^{n M}\end{array}\right]^{T}$ and the $\mathrm{MG}_{n}$ output can be considered as $\boldsymbol{Y}_{M G}^{n}=\left[\Delta \omega_{c o m}^{n} \Delta \boldsymbol{v}_{p c c, d q}^{n} \boldsymbol{Y}_{S C}^{n, p i n}\right]^{T}$. Finally, the matrices can be numerically calculated employing the Robust Control Toolbox functions presented in Section 2.1.

\subsection{Modeling of interlinking lines}

Interlinking lines are also modeled as series RL branches as shown in Fig. 1. The equations are assumed to be expressed in the CRF. Since the interlinking line dynamics are completely similar to the dynamics of the MG network lines, (14) can be rewritten with accurate variables for $\mathrm{IL}_{n l}$ as follows:

$\dot{\boldsymbol{X}}_{I L}^{n l}=\boldsymbol{A}_{I L}^{n l} \boldsymbol{X}_{I L}^{n l}+\boldsymbol{B}_{I L}^{n l} \boldsymbol{U}_{I L}^{n l}$,

$\boldsymbol{Y}_{I L}^{n l}=\boldsymbol{C}_{I L}^{n l} \boldsymbol{X}_{I L}^{n l}$,

where $\boldsymbol{U}_{I L}^{n l}=\left[\Delta \boldsymbol{v}_{p c c, d q}^{n} \Delta \boldsymbol{v}_{p c c, d q}^{l} \Delta \omega_{c o m}^{n}\right]^{T}, \boldsymbol{X}_{I L}^{n l}=\boldsymbol{Y}_{I L}^{n l}=\Delta \boldsymbol{i}_{I L, d q}^{n l}$, and the matrices can be simply calculated.

\subsection{Overall model of interconnected AC microgrids}

By modeling all IMG modules including AC MGs and interlinking lines separately, small-signal modeling of various structures of IMGs is possible. Fig. 5 indicates the details of interconnections among the IMGs shown in Fig. 1. Note that the CRF zone consists of all interlinking lines and it is extended to the MG power part excluding the DER power part. The PCC voltage of each MG is as an input for each interlinking line to this MG. Inversely, the interlinking line current is as the input of the corresponding MGs. Thus, the overall model, which is input-free, is represented in the state space form as:

$\dot{\boldsymbol{X}}_{I M G}=\boldsymbol{A}_{I M G} \boldsymbol{X}_{I M G}$,

where the integrated state vector of the main modules is as:

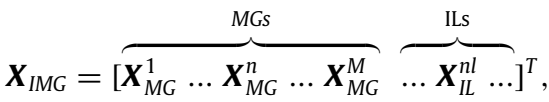

including $(15 m+2) n+2 p$ state variables, where $n$ and $p$ are the number of MGs and interlinking lines, respectively. General form of $\boldsymbol{A}_{I M G}$ is as:

$\mathbf{A}_{I M G}=\left[\begin{array}{cc}\mathbf{A}_{M G S} & \mathbf{A}_{M G, I L} \\ \mathbf{A}_{I L, M G} & \mathbf{A}_{I L S}\end{array}\right]$ where

$$
\begin{aligned}
\mathbf{A}_{M G s}= & {\left[\begin{array}{ccc}
\mathbf{A}_{M G}^{1} & \ldots & \mathbf{B}_{S C}^{1 n} \\
\vdots & \ddots & \vdots \\
\mathbf{B}_{S C}^{n 1} & \cdots & \mathbf{A}_{M G}^{n}
\end{array}\right], \mathbf{A}_{M G, I L}=\left[\begin{array}{ccc}
\mathbf{B}_{M G 1}^{I L 1} & \cdots & \mathbf{B}_{M G 1}^{I L p} \\
\vdots & \ddots & \vdots \\
\mathbf{B}_{M G n}^{I L 1} & \cdots & \mathbf{B}_{M G n}^{I L p}
\end{array}\right], } \\
\mathbf{A}_{I L, M G}= & {\left[\begin{array}{ccc}
\mathbf{B}_{I L 1}^{M G 1} & \ldots & \mathbf{B}_{I L 1}^{M G n} \\
\vdots & \ddots & \vdots \\
\mathbf{B}_{I L p}^{M G 1} & \cdots & \mathbf{B}_{I L p}^{M G n}
\end{array}\right], \mathbf{A}_{I L s}=\left[\begin{array}{ccc}
\mathbf{A}_{I L}^{1} & \cdots & 0 \\
\vdots & \ddots & \vdots \\
0 & \cdots & \mathbf{A}_{I L}^{p}
\end{array}\right] . }
\end{aligned}
$$

$\mathbf{A}_{M G}^{n}$ is given in (18) and it is always nonzero. $\mathbf{B}_{S C}^{1 n}$ shows the secondary control communication structure from $\mathrm{MG}_{1}$ to $\mathrm{MG}_{n}$, which is obtained from $\mathbf{B}_{S C 2}$ in (7). It is nonzero if and only if there exists a communication link between these two MGs. $\mathbf{B}_{M G n}^{I L p}$ indicates interconnections from $\mathrm{MG}_{n}$ to $\mathrm{IL}_{p}$ state variables, which is obtained as a sub-matrix of $\mathbf{B}_{M G}^{n}$ in (18), and it is nonzero if and only if the $\mathrm{MG}_{n}$ is connected to another MG through the $\mathrm{IL}_{p}$. $\mathbf{B}_{I L p}^{M G n}$ indicates interconnections from $\mathrm{IL}_{p}$ to $\mathrm{MG}_{n}$ state variables, which is obtained as a sub-matrix of the typical $\mathbf{B}_{I L}^{n l}$ in (19), and it is nonzero if and only if $\mathbf{B}_{M G n}^{I L p} \neq 0$. Finally, $\mathbf{A}_{I L}^{p}$ is the same with typical $\mathbf{A}_{I L}^{n l}$ in (18). For the typical four-IMGs case shown in Fig. 6, $\mathbf{A}_{4-\mathrm{IMG}}$ is obtained as follows:

$\mathbf{A}_{4-I M G}=\left[\begin{array}{cccc|ccc}\mathbf{A}_{M G}^{1} & 0 & 0 & \mathbf{B}_{S C}^{14} & \mathbf{B}_{M G 1}^{I L 12} & \mathbf{B}_{M G 1}^{I L 14} & 0 \\ 0 & \mathbf{A}_{M G}^{2} & \mathbf{B}_{S C}^{23} & 0 & \mathbf{B}_{M G 2}^{I L 12} & 0 & 0 \\ 0 & \mathbf{B}_{S C}^{32} & \mathbf{A}_{M G}^{3} & \mathbf{B}_{S C}^{34} & 0 & 0 & \mathbf{B}_{M G 3}^{I L 34} \\ \mathbf{B}_{S C}^{41} & 0 & \mathbf{B}_{S C}^{43} & \mathbf{A}_{M G}^{4} & 0 & \mathbf{B}_{M G 4}^{I L 14} & \mathbf{B}_{M G 4}^{I L 34} \\ & & & & & & \\ \hline & & & & & & \\ \mathbf{B}_{I L 12}^{M G 1} & \mathbf{B}_{I L 12}^{M G 2} & 0 & 0 & \mathbf{A}_{I L}^{12} & 0 & 0 \\ \mathbf{B}_{I L 14}^{M G 1} & 0 & 0 & \mathbf{B}_{I L 14}^{M G 4} & 0 & \mathbf{A}_{I L}^{14} & 0 \\ 0 & 0 & \mathbf{B}_{I L 34}^{M G 3} & \mathbf{B}_{I L 34}^{M G 4} & 0 & 0 & \mathbf{A}_{I L}^{34}\end{array}\right]$.

Therefore, the overall IMG state matrix $\boldsymbol{A}_{I M G}$ can be achieved analytically using (22) and (23). Nevertheless, it is easier to be calculated numerically for interconnecting a desired number of the modules using the valuable Robust Control Toolbox functions in MATLAB. Note that the main requirement in this method is to exactly specify the interconnections in each IMG case study as generally shown in Fig. 5.

\section{Prony analysis-based model validation}

In this section, the Prony method as a signal analyzer tool is presented. In addition, the participation matrix used to find the contribution of dynamic modes in the state variables are reviewed. Finally, the obtained model is validated using eigenvalue and Prony analyses. 


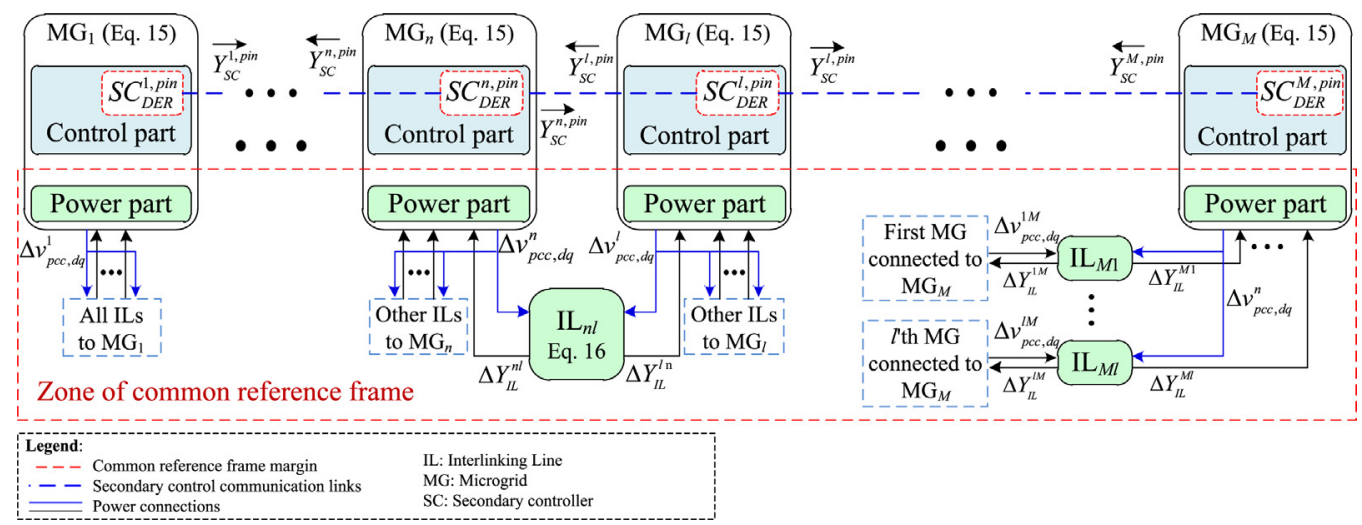

Fig. 5. Specifying interconnections among synchronous microgrids through interlinking lines including all inputs and outputs of the main modules.

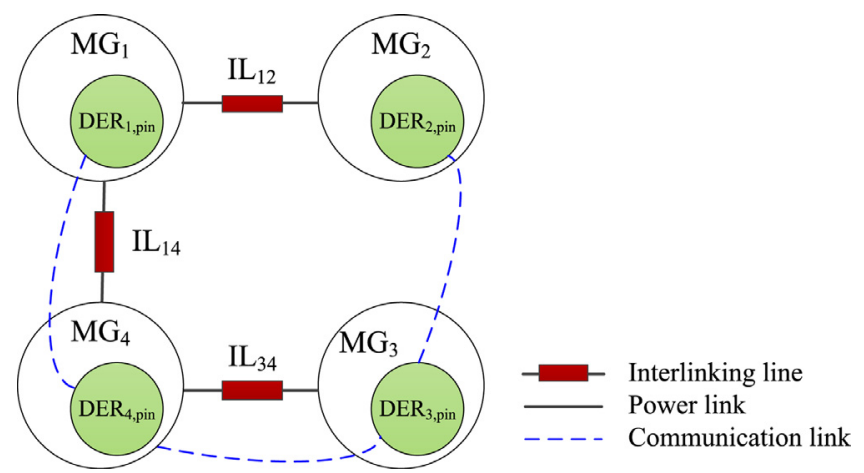

Fig. 6. A typical system of four interconnected microgrids via three interlinking lines and an inter-MG communication network.

\subsection{Prony analysis method}

A signal $y(t)$, which is sampled with the sampling time $T$, can be represented as follows:

$y[k]=\sum_{i=1}^{L} C_{i} \mu_{i}^{k}$,

where $\mathrm{L}$ is total number of estimated damped sinusoid components and $k=0,1, \ldots, N-1$. Unknown parameters $C_{i}$ and $\mu_{i}^{k}$ can be found by comparing (24) with the estimated signal $\hat{y}(t)$, which is constructed by the sum of complex damped sinusoids as follows (Papadopoulos et al., 2014; Golpîra et al., 2015):

$\hat{y}(t)=\sum_{i=1}^{L} A_{i} e^{\sigma_{i}(t)} \cos \left(2 \pi f_{i} t+\phi_{i}\right)$,

where $A_{i}, \sigma_{i}, f_{i}$ and $\phi_{i}$ are amplitude, damping coefficient, frequency and phase of $i$ 'th component. Therefore, $C_{i}$ and $\mu_{i}^{k}$ have following relationships with the parameters of $(25)$ :

$$
\begin{aligned}
C_{i} & =\frac{A_{i}}{2} e^{j \phi_{i}}, \\
\mu_{i}^{k} & =e^{\left(\sigma_{i}+j 2 \pi f_{i}\right) T} .
\end{aligned}
$$

The original Prony analysis computes $C_{i}$ and $\mu_{i}^{k}$ in the three following steps using the samples $y[k]$, such that $k=L, L+$ $1, L+2, \ldots, N-1$ (Zieliński and Duda, 2011; Hauer, 1991; Papadopoulos et al., 2014).

\subsubsection{Predicting linear model}

A linear prediction model can be given by the observed data set as follows:

$y[k]=a_{1} y[k-1]+a_{2} y[k-2]+\cdots+a_{L} y[k-L]$.

In order to obtain $a_{i}$ coefficients, (27) is written for all values of $k$ and forms an equation set, which are represented in a matrix form as follows:

$\mathbf{A}=\mathbf{D}^{+} \mathbf{Y}$

where, $\mathbf{D}^{+}$means the pseudo inverse of $\mathbf{D}, \mathbf{A}=$ $\left[\begin{array}{llll}a_{1} & a_{2} & \cdots & a_{L}\end{array}\right]^{T}, \mathbf{Y}=[y[L] \quad y[L+1] \quad \cdots \quad y[N-1]]^{T}$ and

$\mathbf{D}=\left[\begin{array}{cccc}y[L-1] & y[L-2] & \cdots & y[0] \\ y[L] & y[L-1] & \cdots & y[1] \\ \vdots & \vdots & \vdots & \vdots \\ y[N-2] & y[N-3] & \cdots & y[N-L-1]\end{array}\right]$.

\subsubsection{Root calculation of the predicted model}

The roots of following characteristic polynomial, which is formed from the linear prediction coefficients $a_{i}$ can be found easily using many computation softwares, e.g. MATLAB.

$$
\begin{array}{r}
\mu^{L}-a_{1} \mu^{L-1}-\cdots-a_{L-1} \mu-a_{L}= \\
\left(\mu-\hat{\mu}_{1}\right)\left(\mu-\hat{\mu}_{2}\right) \ldots\left(\mu-\hat{\mu}_{L}\right),
\end{array}
$$

where, $\hat{\mu}_{i}$ depicts the roots of the polynomial.

\subsubsection{Coefficient calculation}

$C_{i}$ coefficients are calculated by substituting $\hat{\mu}_{i}$ in (24) as follows:

$\mathbf{C}=\mathbf{U}^{+} \mathbf{Y}$

where, $\mathbf{C}=\left[\begin{array}{llll}C_{1} & C_{2} & \cdots & C_{L}\end{array}\right]^{T}$ and $\mathbf{U}^{+}$means the pseudo inverse of $\mathbf{U}$, which $\mathbf{U}$ is as follows:

$\mathbf{U}=\left[\begin{array}{cccc}1 & 1 & \cdots & 1 \\ \hat{\mu}_{1} & \hat{\mu}_{2} & \cdots & \hat{\mu}_{L} \\ \vdots & \vdots & \vdots & \vdots \\ \hat{\mu}_{1}^{N-1} & \hat{\mu}_{2}^{N-1} & \cdots & \hat{\mu}_{L}^{N-1}\end{array}\right]$

Finally $A_{i}, \sigma_{i}, f_{i}$ and $\phi_{i}$ can be calculated only by decoupling the real and imaginary components of both sides of (26a) and (26b).

\subsection{Participation matrix}

In order to analyze small-signal stability of the proposed model, the eigenvalues are calculated as follows:

$\left|s \boldsymbol{I}-\boldsymbol{A}_{I M G}\right|=0$, 
Table 2

Power and control information of two interconnected AC microgrids.

\begin{tabular}{|c|c|c|c|c|}
\hline Parameter & \multicolumn{4}{|c|}{ Value } \\
\hline RMS line-line voltage (V) & \multicolumn{4}{|l|}{400} \\
\hline DC voltage $(\mathrm{V})$ & \multicolumn{4}{|l|}{780} \\
\hline Angular frequency (rad/s) & \multicolumn{4}{|c|}{$100 \pi$} \\
\hline $\begin{array}{l}\text { Interlinking line impedance } \\
(\Omega)\end{array}$ & \multicolumn{4}{|c|}{$3.6+\mathrm{j} 1.23$} \\
\hline MGs & \multicolumn{2}{|l|}{$\mathrm{MG}_{1}$} & \multicolumn{2}{|l|}{$\mathrm{MG}_{2}$} \\
\hline MG $P_{\max }(\mathrm{kW})$ & \multicolumn{2}{|l|}{3} & \multicolumn{2}{|l|}{3} \\
\hline MG $Q_{\max }(\mathrm{kVAr})$ & \multicolumn{2}{|l|}{1.5} & \multicolumn{2}{|l|}{1.5} \\
\hline Initial load & \multicolumn{2}{|c|}{$\begin{array}{l}0.6 P_{\max } \\
0.6 Q_{\max }\end{array}$} & \multicolumn{2}{|c|}{$\begin{array}{l}0.5 P_{\max } \\
0.5 Q_{\max }\end{array}$} \\
\hline Controller coefficients & \multicolumn{2}{|l|}{$K_{P}$} & \multicolumn{2}{|l|}{$K_{I}$} \\
\hline PI voltage controller & \multicolumn{2}{|l|}{1} & \multicolumn{2}{|l|}{200} \\
\hline DGs & $\mathrm{DG}_{1}$ & $\mathrm{DG}_{2}$ & $\mathrm{DG}_{3}$ & $\mathrm{DG}_{4}$ \\
\hline DG ratings $\left(S_{\max }\right)(\mathrm{kVA})$ & 1.12 & 2.24 & 1.12 & 2.24 \\
\hline $\begin{array}{l}\text { Series filter inductance } \\
(\mathrm{mH})\end{array}$ & 1.8 & 1.8 & 1.8 & 1.8 \\
\hline Series filter resistance $(\Omega)$ & 0.05 & 0.05 & 0.05 & 0.05 \\
\hline Shunt filter capacitance $(\mu F)$ & 3.7 & 7.5 & 3.7 & 7.5 \\
\hline Line inductance $(\mathrm{mH})$ & 15.6 & 7.8 & 15.6 & 7.8 \\
\hline Line resistance $(\Omega)$ & 3.6 & 1.8 & 3.6 & 1.8 \\
\hline $\begin{array}{l}\text { P-f droop coefficients } \\
\left(10^{-3} \mathrm{rad} / \mathrm{s} \mathrm{W}\right)\end{array}$ & 1.6 & 0.8 & 1.6 & 0.8 \\
\hline $\begin{array}{l}\text { Q-V droop coefficients } \\
\left(10^{-2} \mathrm{~V} / \mathrm{VAR}\right)\end{array}$ & 6.5 & 3.2 & 6.5 & 3.2 \\
\hline $\begin{array}{l}\text { Leader/follower secondary } \\
\text { control delay }(T / \tau)(\mathrm{s})\end{array}$ & 0.2 & 10 & 0.2 & 10 \\
\hline
\end{tabular}

where $s$ is Laplace operator and $I$ is the identity matrix with appropriate size. In this study, the contribution of the eigenvalues in the IMG state variables are required to be compared with the Prony analysis outputs. The participation matrix indicates the contribution of each eigenvalue in each state variable and vice versa, which can be calculated as (Shahnia and Arefi, 2017):

$\boldsymbol{M}_{P F}=\left(\boldsymbol{\Phi}^{-1}\right) \bullet \boldsymbol{\Phi}$,

where $\Phi$ is the right eigenvector matrix of $\boldsymbol{A}_{I M G}$ and $\bullet$ denotes Hadamard product. $\boldsymbol{M}_{P F}(i, j)$ shows the involvement of $i$ 'th state variable in $j$ 'th eigenvector and also the involvement of $j$ 'th eigenvector in $i$ 'th state variable. Thus, in order to find each state variable, all participant eigenvalues should be taken into account. In fact, each participant in $i$ 'th state variable is equivalent to a non-zero element of $i$ 'th row in $\boldsymbol{M}_{P F}$, which is a positive number less than/equal to 1 if the matrix elements (participation factors) are normalized as follows (Shahnia and Arefi, 2017):

$\boldsymbol{M}_{P F, n}(i, j)=\left|\boldsymbol{M}_{P F}(i, j)\right| / \sum_{m} \boldsymbol{M}_{P F}(i, m)$,

where $\boldsymbol{M}_{P F, n}(i, j)$ is the normalized participation share of $j$ 'th eigenvalue in the $i$ 'th state variable.

\subsection{Model validation procedure}

Fig. 7 shows a block diagram of the proposed validation method. The measured waveforms of the real-time simulated IMGs are analyzed by the Prony method. In order to take precise results from the Prony analysis, a perturbed form of the measured waveform must be found by subtracting from the set-point. In addition, the Prony analysis parameters should be chosen carefully including number of damped sinusoids $(L)$, data window width (equivalent to $k$ ) and sampling time $(T)$. As a result, all composing damped sinusoids of the perturbed waveform are estimated in the form of natural frequencies $\left(\gamma_{i}=\sigma_{i} \pm j 2 \pi f_{i}\right)$, amplitudes $\left(A_{i}\right)$ and phases $\left(\phi_{i}\right)$.
In order to realize the proposed validation, the dominant participating eigenvalues in the state variable and their participation factors should be equal or near to the natural frequencies and their corresponding amplitudes of the Prony estimated state variable, respectively. The model validation error (MVE), which shows the similarity percentage, is as (Naderi et al., 2019b):

$M V E_{i}=\frac{\left|\lambda_{i}-\gamma_{i}\right|}{\left|\lambda_{i}\right|} \times 100$

where $\mathrm{MVE}_{i}$ is the relative error percentage that indicates the difference between estimated frequency $\gamma_{i}$ by Prony analysis and correlated eigenvalue $\lambda_{i}$ calculated by eigenvalue analysis. A similar relation can also be used for the difference between perunit contribution in waveform $A_{i}(p u)$ (instead of $\gamma_{i}$ in (34)) and correlated normalized participation factor $M_{P F, n}$ (instead on $\lambda_{i}$ in (34)). Note that the values are per-unit in this case. Therefore, a relative error for $M_{V E}$ is not required.

\section{Real-time simulation results}

In order to verify the proposed modeling method, a typical two IMGs presented in Naderi et al. (2019a), which is based on the general structure shown in Fig. 1 is implemented in an OPAL-RT simulator. Table 2 comprises the electrical and control parameters used to find the numerical form of $A_{I M G}$ for the two IMGs. Moreover, initial values are obtained from the real-time simulation of the IMGs based on the information of Table 2 .

\subsection{OPAL-RT simulator}

The simulations are done in OPAL-RT simulator OP5600 to show the ability of working the study IMGs in real time. Fig. 8(a) shows the experimental setup including the host PC, the OPALRT target and a LAN cable for networking. Fig. 8(b) shows the conceptual diagram of the real-time simulation process. RT-LAB software is used as the interface between MATLAB and realtime OPAL-RT simulator. The MATLAB/SimPowerSystems model is loaded on the OPAL-RT through the RT-LAB and the real-time data is come back to MATLAB conversely. Note that the model is split into two subsystems, i.e. a subsystem including all permanent power and control parts during the real-time simulation and a subsystem including real-time displays and changeable parameters.

\subsection{Eigenvalue analysis results}

Fig. 9 shows all eigenvalues of the proposed model for two IMGs, where four different frequency ranges can be observed. Very far eigenvalues from the imaginary axis are related to the DER power part, i.e. RLC filters (Group 1). The eigenvalues around -600 real correlate to the MG loads, power network, interlinking line and somewhat inner controllers (Group 2). The eigenvalues around -200 real are generally related to the inner controllers (Group 3). The dominant modes are mostly related to the secondary and primary controllers as well as DER coupling lines (Group 4).

\subsection{Prony analysis results}

Fig. 10 shows the perturbed form of three measured waveforms from the real-time simulated IMGs and their estimation using the Prony analysis, which is applied at the time interval of $0 \mathrm{~s}$ to $1 \mathrm{~s}$ i.e $k . T=1 \mathrm{~s}$. For a precise estimation in each case, $L$ and $T$ are changed and the convenient values are found by trial and error method, which are indicated in Table 3. $\mathrm{MG}_{1}$ and $\mathrm{MG}_{2}$ are operated in $60 \%$ and $50 \%$ of their nominal load, 


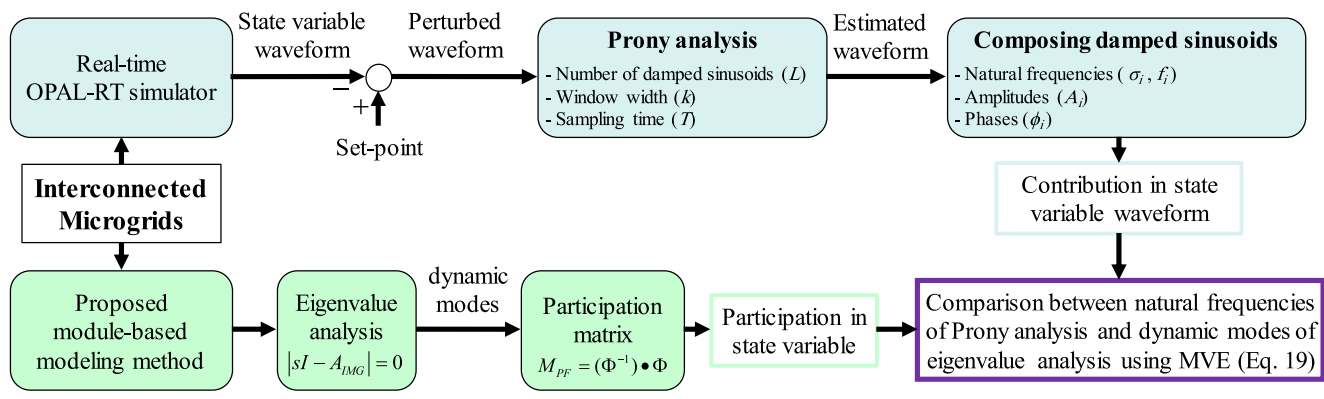

Fig. 7. Block diagram of the proposed model validation by comparing the outputs of the eigenvalue and Prony analyses.

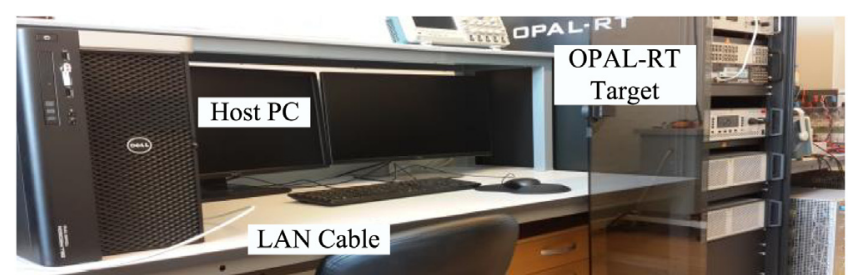

(a)

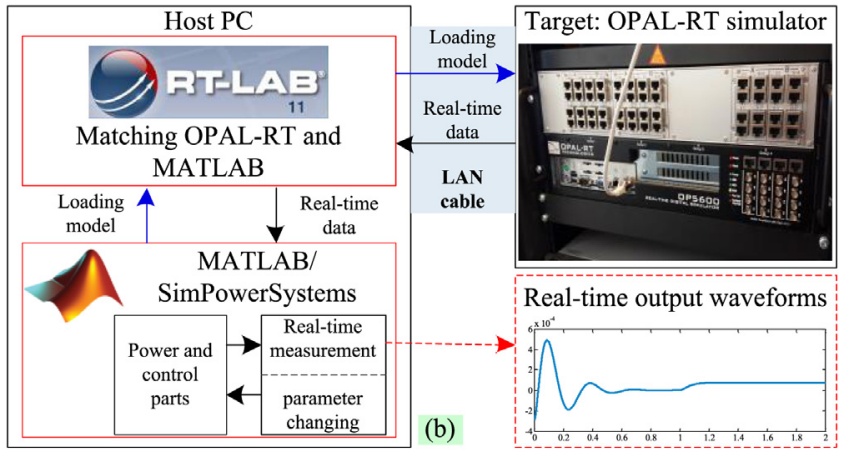

Fig. 8. (a) Real-time setup including the host PC, the OPAL-RT target and a LAN cable. (b) Conceptual diagram of the real-time simulation process.

respectively. As the second set-point, the $\mathrm{MG}_{1}$ load increases to $90 \%$ of its nominal value at $t=1 \mathrm{~s}$. This recent change shows only the different dynamic responses of two different setpoints and initial conditions. Note that a state variable waveform can show all participating natural frequencies only when the input or the initial condition is applied in the direction of all its eigenvectors. In this situation, the Prony analysis can correctly estimate all natural frequencies. By comparing several initial conditions, the best identification can be achieved by the zero initial condition.

According to Fig. 10, $\Delta I_{I L d}, \Delta f_{2}^{M G 2}$ and $\Delta \delta_{2}^{M G 1}$ are estimated well by the Prony analysis. In fact, the real-time simulated waveform is fitted by the Prony estimated waveform in all three cases.

\subsection{Comparison results}

The Prony estimation results are classified in Table 3 including the natural frequencies and their related contribution in the waveforms. On the other hand, the results of the eigenvalue analysis are depicted in Table 3 including the dynamic modes and their participation in the state variables.

According to the comparison results in all three cases, the natural frequencies and dynamic modes are not exactly the same. However, there is an appropriate similarity between the frequency ranges and even the real and imaginary values in some cases. These similarities are shown by placing similar natural

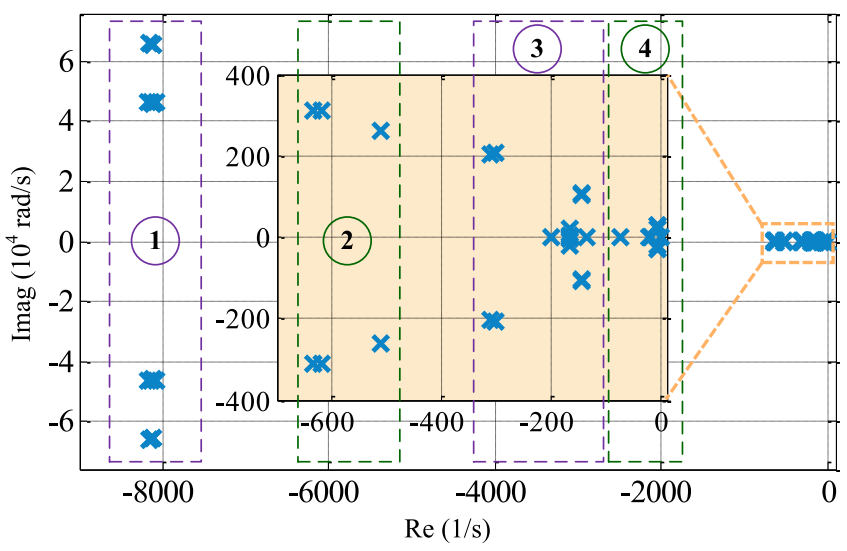

Fig. 9. All eigenvalues of the two interconnected AC microgrids.
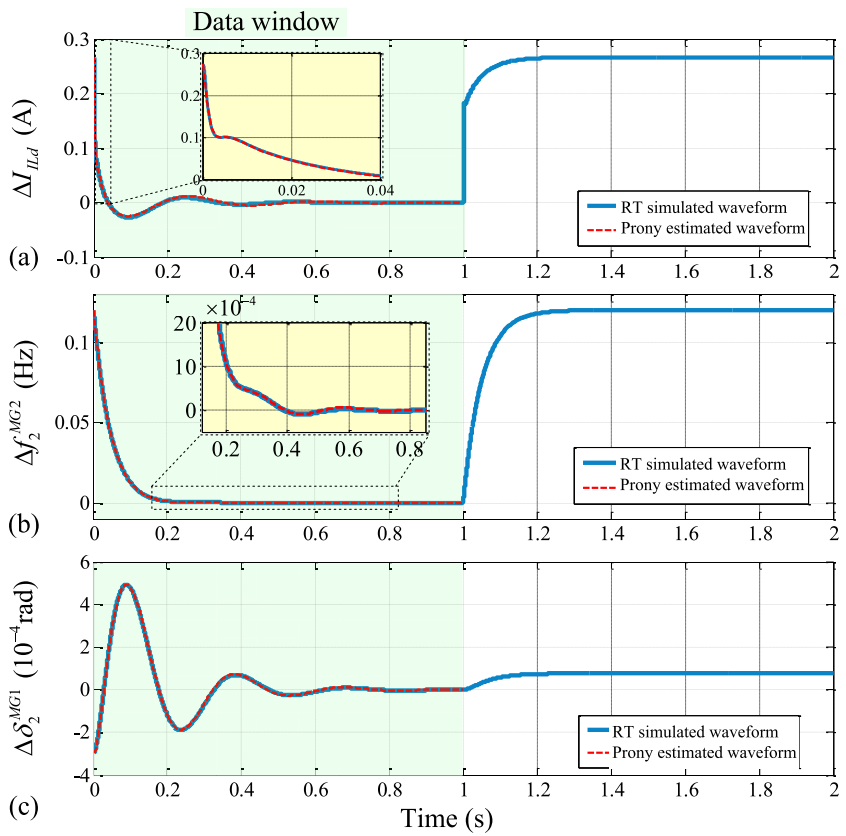

Fig. 10. Simulated waveforms and their Prony estimation: (a) direct component of interlinking line current, (b) frequency of $\mathrm{DER}_{2}$ in $\mathrm{MG}_{2}$, (c) angle difference of $\mathrm{DER}_{2}$ voltage in $\mathrm{MG}_{1}$ from the common reference frame.

frequencies and dynamic modes in same rows. Note that the lack of complete equivalency can be due to deletion of some dynamics during linearization or the lack of exciting some natural frequencies. Moreover, Fig. 11 shows MVE for the listed variables in Table 3 including dynamic modes and their per-unit participation factor (PF in the legend). The third mode of $\Delta I_{I L}$, which 
Table 3

Comparison between outputs of the Prony and eigenvalue analyses.

\begin{tabular}{|c|c|c|c|c|c|c|c|}
\hline \multirow{2}{*}{$\begin{array}{l}\text { State } \\
\text { variable }\end{array}$} & \multirow{2}{*}{$\begin{array}{l}\text { Number of damped } \\
\text { sinusoids } L\end{array}$} & \multirow{2}{*}{$\begin{array}{l}\text { Sampling } \\
\text { time } T(\mathrm{~s})\end{array}$} & \multirow{2}{*}{$\begin{array}{l}\text { Mode } \\
\text { number }\end{array}$} & \multicolumn{2}{|c|}{ Prony analysis outputs } & \multicolumn{2}{|c|}{ Eigenvalues analysis outputs } \\
\hline & & & & $\begin{array}{l}\text { Natural } \\
\text { frequencies }(\gamma)\end{array}$ & $\begin{array}{l}\text { Contribution in } \\
\text { waveform (per-unit) }\end{array}$ & $\overline{\text { Dynamic mode }(\lambda)}$ & $\begin{array}{l}\text { Contribution in state } \\
\text { variable/participation } \\
\text { factors (per-unit) }\end{array}$ \\
\hline \multirow{4}{*}{$\Delta I_{I L}$} & \multirow{4}{*}{6} & \multirow{4}{*}{0.004} & $\begin{array}{l}1 \\
2\end{array}$ & $\begin{array}{l}-4.74 \pm j 18.47 \\
-59.76\end{array}$ & $\begin{array}{l}0.134 \\
0.36\end{array}$ & $\begin{array}{l}-6.75 \pm j 20.95 \\
-73.49\end{array}$ & $\begin{array}{l}0.114 \\
0.33\end{array}$ \\
\hline & & & 3 & $-453.61 \pm j 511.31$ & 0.34 & $\begin{array}{l}-509.69 \pm j 261.80 \\
-635.07 \pm j 312.67\end{array}$ & $\begin{array}{l}0.14 \\
0.132\end{array}$ \\
\hline & & & $\overline{4}$ & -452 & 0.16 & -467 & 0.14 \\
\hline & & & & & & $\begin{array}{l}-166.26 \pm j 19.38 \\
\text { Other }\end{array}$ & $\begin{array}{l}0.137 \\
0.01 \\
\end{array}$ \\
\hline \multirow{4}{*}{$\Delta f_{2}^{M G 2}$} & \multirow{4}{*}{9} & \multirow{4}{*}{0.004} & 1 & $-5.35 \pm j 22.35$ & 0.218 & $\begin{array}{l}-6.75 \pm j 20.95 \\
-8.26 \pm j 28.82\end{array}$ & $\begin{array}{l}0.334 \\
0.206\end{array}$ \\
\hline & & & 2 & -22.40 & 0.531 & $\begin{array}{l}-19.94 \\
-22.12 \\
\end{array}$ & $\begin{array}{l}0.312 \\
0.025 \\
\end{array}$ \\
\hline & & & 3 & -81.22 & 0.175 & -73.49 & 0.122 \\
\hline & & & & $\begin{array}{l}-305.72 \\
-146.69 \pm j 73.18 \\
-605 \pm j 219.91\end{array}$ & $\begin{array}{l}0.002 \\
0.01 \\
0.064\end{array}$ & Other & 0.01 \\
\hline \multirow{3}{*}{$\Delta \delta_{2}^{M G 1}$} & \multirow{3}{*}{6} & \multirow{3}{*}{0.001} & $\begin{array}{l}1 \\
2\end{array}$ & $\begin{array}{l}-6.52 \pm j 21.39 \\
-79.88\end{array}$ & $\begin{array}{l}0.76 \\
0.208\end{array}$ & $\begin{array}{l}-8.27 \pm j 28.68 \\
-73.49\end{array}$ & $\begin{array}{l}0.806 \\
0.152\end{array}$ \\
\hline & & & 3 & $-140.58 \pm j 217.93$ & 0.024 & $\begin{array}{l}-145.17 \pm j 186.91 \\
-143.71 \pm j 192.51\end{array}$ & $\begin{array}{l}0.012 \\
0.016\end{array}$ \\
\hline & & & & -3419.19 & 0.006 & Other & 0.01 \\
\hline
\end{tabular}

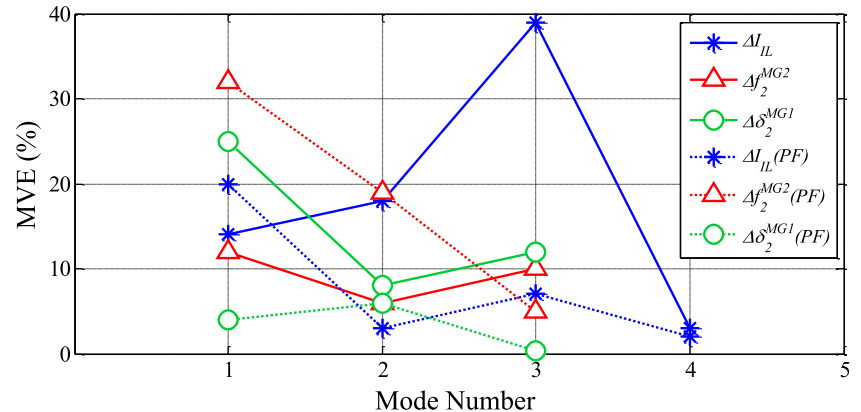

Fig. 11. Model validation error (MVE) for listed variables in Table 3 (solid line for MVE and dotted line for $\mathrm{MVE}_{P F}$ ).

is calculated by averaging, has the maximum MVE. This mode is medium frequency, thus it is considerably affected by the linearization process. The maximum $\mathrm{MVE}_{P F}$ is $32 \%$, which belongs to the first mode of $\Delta f_{2}^{M G 1}$. The low frequency modes, which can be observed in the power controller variables, e.g. $\Delta f_{2}^{M G 2}$ and $\Delta \delta_{2}^{M G 1}$, are more taken into account in the stability studies (Nikolakakos et al., 2017) and generally are validated with MVE less than $20 \%$.

Note that in order to simplify the model and use in stability analysis, one can reduce the main model order to these lowfrequency modes (Naderi et al., 2020a). As it can be seen in the first two rows of $\Delta f_{2}^{M G 2}$ and $\Delta \delta_{2}^{M G 1}$ in Table 3, these low frequency modes are validated with an appropriate MVE shown in Fig. 11. Therefore, an important point is that Prony analysis can be used to find a simplified model of large-scale systems such as IMGs by measuring a remarkable characteristic, e.g. frequency and analyzing it by Prony tool. Such a measurement-based modeling method and correlated data-driven control methods will studied in future works.

\section{Parameters sensitivity analysis}

Figs. 12 and 13 shows the sensitivity analysis of the most dominant parameters of the CB-IMGs, i.e. primary and secondary controllers parameters. In Fig. 12(a) and (b) the $\omega-P$ droop characteristic gains of $\mathrm{MG}_{1}$ 's DERs and $\mathrm{MG}_{2}$ 's DERs are modified within $[0.15] \% .\left(\omega_{n} / P_{\max }^{D E R}\right)$, respectively. The most dominant modes affected by changing $\omega-P$ droop gain $K_{p}$ are shown by $\lambda_{1}$ and $\lambda_{2} . \lambda_{1}$ is very close to the imaginary axis for very low value of $K_{p}$. The modes change to an oscillatory mode by increasing $K_{p}$ and will decrease the stability margin. For, the same criteria for changing the $K_{p}$ gain, $K_{p}^{M G 1}$ increase does not cause instability, however $K_{p}^{M G 2}$ increase leads to instability for $K_{p, D E R 1}^{M G 2}>7.2 \mathrm{rad} / \mathrm{kW} \mathrm{s}$. In Fig. $12(\mathrm{c})$ and (d) the $v-Q$ droop characteristic gains of $\mathrm{MG}_{1}$ 's DERs and $\mathrm{MG}_{2}$ 's DERs are changed within [1 15$] \%$. $\left(v_{n} / Q_{\max }^{D E R}\right)$, respectively. Increasing the $K_{q}$ for both MGs results in the stability margin enhancement. Note that the nearest eigenvalues to the imaginary axis are not affected by the $K_{q}$ gain modifications. Moreover, there exist small changes of the oscillatory modes, especially in Fig. 12(d) for changes of the $\mathrm{MG}_{2}$ $v-Q$ droop gains. However, in Fig. 12(c), the frequency of the dominant oscillatory modes increases as $4 \mathrm{~Hz}$.

According to Fig. 13(a) and (b), when both the secondary controller parameters $T$ and $\tau$ increase, the most dominant eigenvalue moves from the very close position to the imaginary axis to the left side, where the stability margin is improved. Other dominant real modes in Fig. 13(a) have a same behavior for the $T$ change. Nevertheless, the dominant oscillatory modes have negligible movement for the $T$ change. On the other hand, the dominant oscillatory modes have considerable changes in their real value for modifying the $\tau$ as shown in Fig. 13(b).

\section{Conclusion}

This paper proposes a comprehensive, generalized, and expandable small-signal model of synchronous interconnected AC 

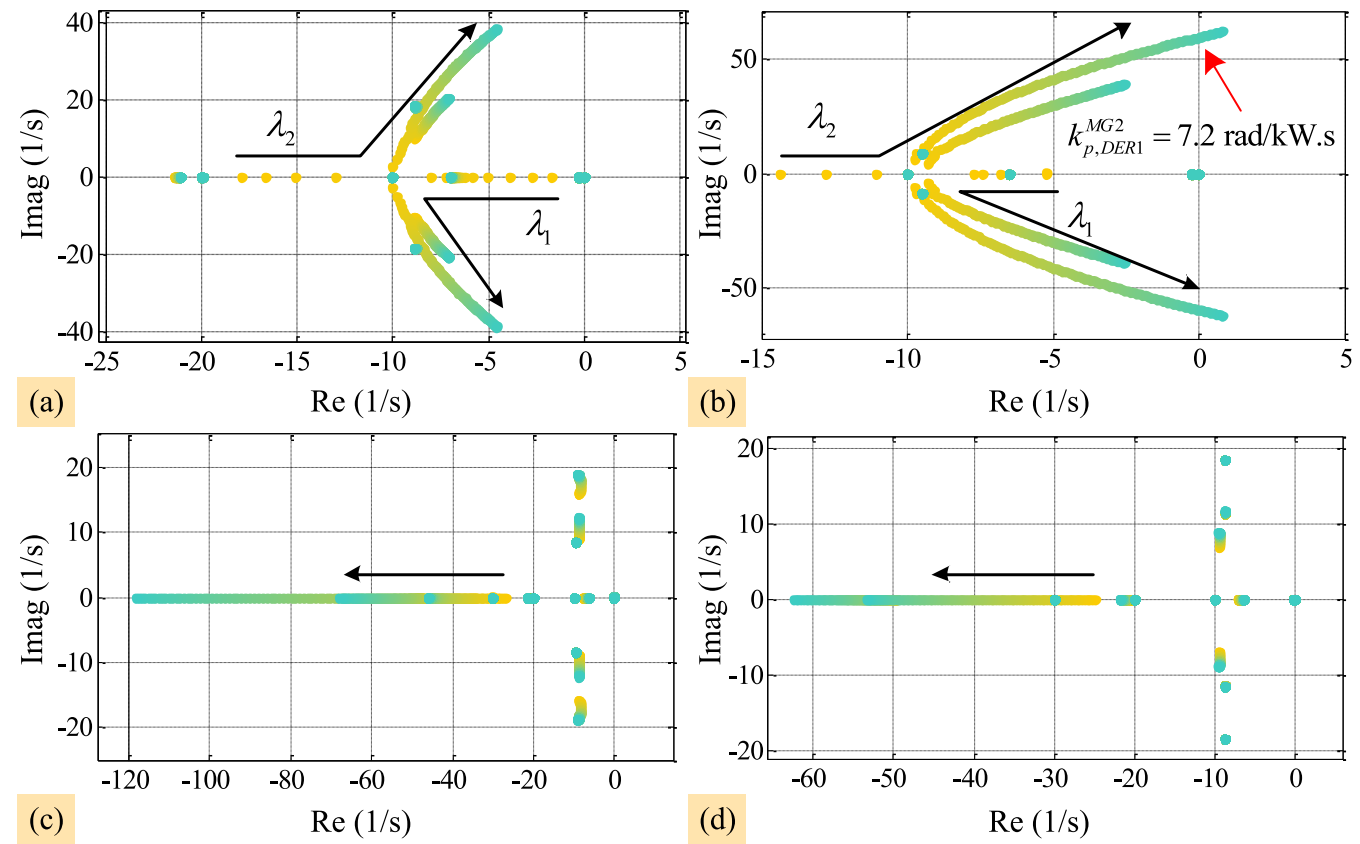

Fig. 12. Sensitivity analysis output for the primary controller parameters: (a) $K_{p}^{M G 1} \in[0.15] \%$. $\left(\omega_{n} / P_{\max }^{D E R}\right),\left(\right.$ b) $K_{p}^{M G 2} \in[0.15] \% .\left(\omega_{n} / P_{\max }^{D E R}\right),\left(\right.$ c) $K_{q}^{M G 1} \in[115] \%$.( $\left.v_{n} / Q_{\max }^{D E R}\right)$, (d) $K_{q}^{M G 2} \in[115] \% .\left(v_{n} / Q_{\max }^{D E R}\right)$.
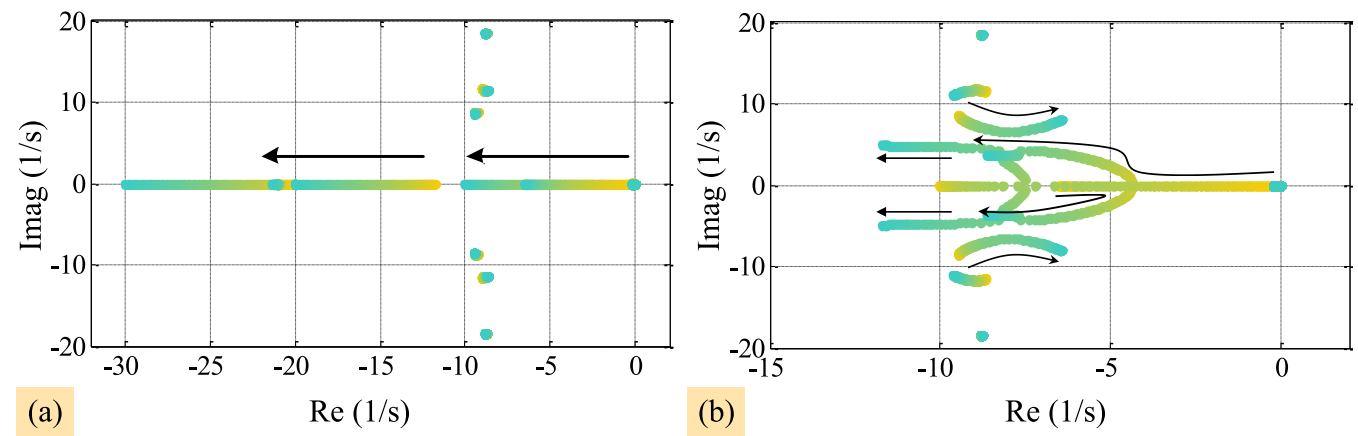

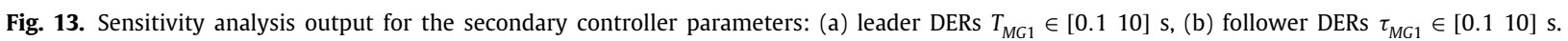

microgrids together with a standard model validation approach. In the module-based small-signal modeling method, the standard state space representation is used for each module and modules interconnections either are found analytically or are calculated using powerful Robust Control Toolbox functions in MATLAB. Prony and eigenvalue analyses as well as participation matrix are used as powerful instruments to validate the proposed model. Using Prony analysis, the measured waveforms of real-time simulated interconnected microgrids are estimated with a sum of damped sinusoids. Then, their natural frequencies and amplitudes are compared with the dynamic modes of the model and their participation in the state variables, which are calculated using eigenvalue analysis and participation matrix, respectively. The results exhibit that Prony analysis can estimate the real-time simulated waveforms accurately. Moreover, the model validation error is less than $20 \%$ in most of the cases. Nevertheless, in comparison, some results of the two analyses are not completely the same due to the lack of excitation of some frequencies or their deletion during linearization. Therefore, Prony analysis can be used to validate the small-signal model. Furthermore, the small-signal stability is analyzed using eigenvalue and sensitivity analyses, which shows a considerable impact of the secondary and $\omega-P$ primary control parameters on the stability margins.
One can conclude that a model can be found for large-scale systems such as interconnected microgrids, especially when the model focus on a specific characteristic, e.g. frequency, which will left for future works.

\section{CRediT authorship contribution statement}

Mobin Naderi: Conceptualization, Methodology, Software, Investigation, Writing - original draft. Yousef Khayat: Conceptualization, Methodology, Resources, Writing - original draft. Qobad Shafiee: Conceptualization, Writing - review \& editing, Visualization. Tomislav Dragicevic: Software, Data curation, Validation. Hassan Bevrani: Methodology, Writing - review \& editing, Supervision. Frede Blaabjerg: Writing - review \& editing, Supervision, Project administration.

\section{Declaration of competing interest}

The authors declare that they have no known competing financial interests or personal relationships that could have appeared to influence the work reported in this paper. 


\section{Acknowledgments}

The research is funded by a Villum Investigator grant (no. 25920) at the Department of Energy Technology, Aalborg University as a part of the Villum Investigator Program funded by the Villum Foundation.

\section{References}

Ali, H., Magdy, G., Xu, D., 2021. A new optimal robust controller for frequency stability of interconnected hybrid microgrids considering non-inertia sources and uncertainties. Int. J. Electr. Power Energy Syst. 128, 106651.

Bevrani, H., 2014. Robust Power System Frequency Control. Springer.

Bevrani, H., François, B., Ise, T., 2017. Microgrid Dynamics and Control. John Wiley \& Sons.

Bevrani, H., Watanabe, M., Mitani, Y., 2014. Power System Monitoring and Control. John Wiley \& Sons.

Bracale, A., Caramia, P., Carpinelli, G., 2007. Adaptive prony method for waveform distortion detection in power systems. Int. J. Electr. Power Energy Syst. 29 (5), 371-379.

de la O Serna, J.A., Ramirez, J.M., Mendez, A.Z., Paternina, M.R.A., 2016. Identification of electromechanical modes based on the digital Taylor-Fourier transform. IEEE Trans. Power Syst. 31 (1), 206-215.

Dong, C., et al., 2018. Time-delay stability switching boundary determination for DC microgrid clusters with the distributed control framework. Appl. Energy 228, 189-204.

Dragicevic, T., Zheng, C., Rodriguez, J., Blaabjerg, F., 2019. Robust quasi-predictive control of LCL-filtered grid converters. IEEE Trans. Power Electron. http: //dx.doi.org/10.1109/TPEL.2019.2916604.

Ferdowsi, F., Vahedi, H., Abianeh, A.J., Edrington, C.S., Elmezyani, T., 2022. A data-driven real-time stability metric for SST-based microgrids. Int. J. Electr. Power Energy Syst. 134, 107397.

Føyen, S., Kvammen, M.-E., Fosso, O.B., 2018. Prony's method as a tool for power system identification in smart grids. In: IEEE Int. Symp. Power Electron., Electr. Drives, Autom. and Motion (SPEEDAM). pp. 562-569.

Golpîra, H., Haghifam, M.R., Seifi, H., 2015. Dynamic power system equivalence considering distributed energy resources using prony analysis. Int. Trans. Electr. Energy Syst. 25 (8), 1539-1551.

Golsorkhi, M.S., Hill, D.J., Karshenas, H.R., 2018. Distributed voltage control and power management of networked microgrids. IEEE J. Emerg. Sel. Top. Power Electron. 6 (4), 1892-1902.

Han, R., Tucci, M., Martinelli, A., Guerrero, J.M., Ferrari-Trecate, G., 2018. Stability analysis of primary plug-and-play and secondary leader-based controllers for DC microgrid clusters. IEEE Trans. Power Syst. 34 (3), 1780-1800.

Hao, R., Ai, Q., Jiang, Z., Zhu, Y., 2018. A novel adaptive control strategy of interconnected microgrids for delay-dependent stability enhancement. Int. J. Electr. Power Energy Syst. 99, 566-576.

Hauer, J., 1991. Application of prony analysis to the determination of modal content and equivalent models for measured power system response. IEEE Trans. Power Syst. 6 (3), 1062-1068.

He, J., Wu, X., Wu, X., Xu, Y., Guerrero, J.M., 2019. Small-signal stability analysis and optimal parameters design of microgrid clusters. IEEE Access 7, 36896-36909.

Hirase, Y., Ohara, Y., Bevrani, H., 2020. Virtual synchronous generator based frequency control in interconnected microgrids. Energy Rep. 6, 97-103.

Hossain, M.J., Mahmud, M.A., Milano, F., Bacha, S., Hably, A., 2016. Design of robust distributed control for interconnected microgrids. IEEE Trans. Smart Grid 7 (6), 2724-2735.

Jiang, S., Annakkage, U., Gole, A., 2006. A platform for validation of FACTS models. IEEE Trans. Power Deliv. 21 (1), 484-491.

Ju, Y., Gao, Z., Li, Z., Chen, X., Zhen, J., 2021. A review on transient stability of land-sea networked fishery microgrids. Inf. Process. Agric..

Khayat, Y., et al., 2019. On the secondary control architectures of AC microgrids: An overview. IEEE Trans. Power Electron. 35 (6), 6482-6500.

Lai, J., Lu, X., Yu, X., Monti, A., 2019. Cluster-oriented distributed cooperative control for multiple AC microgrids. IEEE Trans. Ind. Inf. 15 (11), 5906-5918.

Lasseter, R.H., Jun. 2011. Smart distribution: Coupled microgrids. Proc. IEEE 99 (6), 1074-1082.

Liu, W., Gu, W., Xu, Y., Wang, Y., Zhang, K., 2017. General distributed secondary control for multi-microgrids with both PQ-controlled and droop-controlled distributed generators. IET Gener. Transm. Distrib. 11 (3), 707-718.

Lu, X., Lai, J., Yu, X., 2020. A novel secondary power management strategy for multiple AC microgrids with cluster-oriented two-layer cooperative framework. IEEE Trans. Ind. Inf. 17 (2), 1483-1495.

Majumder, R., Bag, G., 2014. Parallel operation of converter interfaced multiple microgrids. Int. J. Electr. Power Energy Syst. 55, 486-496.

Mudaliyar, S., Duggal, B., Mishra, S., 2020. Distributed tie-line power flow control of autonomous DC microgrid clusters. IEEE Trans. Power Electron. 35 (10), 11250-11266.
Naderi, M., Khayat, Y., Heydari, R., Shafiee, Q., Dragicevic, T., Bevrani, H., Blaabjerg, F., 2019a. Model validation of power electronics-based networked micro-grids by prony analysis. In: 2019 21th Euro. Conf. Power Electron. and Appl. (EPE'19 ECCE Europe). IEEE, pp. 1-9.

Naderi, M., Khayat, Y., Shafiee, Q., Dragicevic, T., Bevrani, H., Blaabjerg, F., 2019b. Interconnected autonomous ac microgrids via back-to-back converters-Part I: Small-signal modeling. IEEE Trans. Power Electron. 35 (5), 4728-4740.

Naderi, M., Shafiee, Q., Bevrani, H., Blaabjerg, F., 2020a. Low-frequency smallsignal modeling of interconnected AC microgrids. IEEE Trans. Power Syst. 36 (4), 2786-2797.

Naderi, M., et al., 2020b. Interconnected autonomous AC microgrids via backto-back converters-Part II: Stability analysis. IEEE Trans. Power Electron. 35 (11), 11801-11812.

Nikolakakos, I.P., Zeineldin, H.H., El-Moursi, M.S., Hatziargyriou, N.D., 2016. Stability evaluation of interconnected multi-inverter microgrids through critical clusters. IEEE Trans. Power Syst. 31 (4), 3060-3072.

Nikolakakos, I.P., Zeineldin, H., El-Moursi, M.S., Kirtley, J.L., 2017. Reduced-order model for inter-inverter oscillations in islanded droop-controlled microgrids. IEEE Trans. Smart Grid 9 (5), 4953-4963.

Papadopoulos, P.N., Papadopoulos, T.A., Crolla, P., Roscoe, A.J., Papagiannis, G.K., Burt, G.M., 2014. Measurement-based analysis of the dynamic performance of microgrids using system identification techniques. IET Gener. Transm. Distrib. 9 (1), 90-103.

Pogaku, N., Prodanovic, M., Green, T.C., 2007. Modeling, analysis and testing of autonomous operation of an inverter-based microgrid. IEEE Trans. Power Electron. 22 (2), 613-625.

Rehimi, S., Mirzaei, R., Bevrani, H., 2020. Interconnected microgrids frequency response model: An inertia-based approach. Energy Rep. 6, 179-186.

Ren, L., et al., 2018. Enabling resilient distributed power sharing in networked microgrids through software defined networking. Appl. Energy 210, 1251- 1265 .

Sahoo, S., Mishra, S., Fazeli, S.M., Li, F., Dragičević, T., 2019. A distributed fixedtime secondary controller for DC microgrid clusters. IEEE Trans. Energy Convers. 34 (4), 1997-2007.

Sauer, P.W., Pai, M.A., Chow, J.H., 2017. Power System Dynamics and Stability: With Synchrophasor Measurement and Power System Toolbox. John Wiley \& Sons.

Serna, J.A., 2013. Synchrophasor estimation using prony's method. IEEE Trans. Instrum. Meas. 62 (8), 2119-2128.

Shahidehpour, M., Li, Z., Bahramirad, S., Li, Z., Tian, W., 2017. Networked microgrids: Exploring the possibilities of the IIT-bronzeville grid. IEEE Power Energy Mag. 15 (4), 63-71.

Shahnia, F., 2016. Stability and eigenanalysis of a sustainable remote area microgrid with a transforming structure. Sustain. Energy Grids Netw. 8, 37-50.

Shahnia, F., Arefi, A., 2017. Eigenanalysis-based small signal stability of the system of coupled sustainable microgrids. Int. J. Electr. Power Energy Syst. 91, 42-60.

Shuai, Z., Peng, Y., Liu, X., Li, Z., Guerrero, J.M., Shen, Z.J., 2018. Dynamic equivalent modeling for multi-microgrid based on structure preservation method. IEEE Trans. Smart Grid http://dx.doi.org/10.1109/TSG.2018.2844107.

Sinha, S., Ghosh, S., Bajpai, P., 2021. Power sharing through interlinking converters in adaptive droop controlled multiple microgrid system. Int. J. Electr. Power Energy Syst. 128, 106649.

TP462, Formerly, 2012. Identification of electromechanical modes in power systems. IEEE Task Force Rep..

Weng, S., Xue, Y., Luo, J., Li, Y., 2020. Distributed secondary control for islanded microgrids cluster based on hybrid-triggered mechanisms. Processes 8 (3), 370.

Wu, X., Xu, Y., Wu, X., He, J., Guerrero, J.M., Liu, C.-C., Schneider, K.P., Ton, D.T., 2019. A two-layer distributed control method for islanded networked microgrid systems. IEEE Trans. Smart Grid 11 (2), 942-957.

Yao, W., Wang, Y., Xu, Y., Lin, P., Qi, Y., Wu, Q., 2021. Distributed layered control and stability analysis of islanded networked-microgrids. Int. J. Electr. Power Energy Syst. 129, 106889.

Zamora, R., Srivastava, A.K., 2018. Multi-layer architecture for voltage and frequency control in networked microgrids. IEEE Trans. Smart Grid 9 (3), 2076-2085.

Zhang, Y., Xie, L., Ding, Q., 2016. Interactive control of coupled microgrids for guaranteed system-wide small signal stability. IEEE Trans. Smart Grid 7 (2), 1088-1096.

Zhao, S., Loparo, K.A., 2017. Forward and backward extended prony (FBEP) method for power system small-signal stability analysis. IEEE Trans. Power Syst. 32 (5), 3618-3626.

Zhao, Z., Yang, P., Wang, Y., Xu, Z., Guerrero, J.M., 2017. Dynamic characteristics analysis and stabilization of PV-based multiple microgrid clusters. IEEE Trans. Smart Grid 10 (1), 805-818.

Zieliński, T., Duda, K., 2011. Frequency and damping estimation methods-an overview. Metrol. Meas. Syst. 18 (4), 505-528. 